\title{
Spectral Gaps for Self-Adjoint Second Order Operators
}

\author{
Denis Borisov and Ivan Veselić
}

\begin{abstract}
We consider a second order self-adjoint operator in a domain which can be bounded or unbounded. The boundary is partitioned into two parts with Dirichlet boundary condition on one of them, and Neumann condition on the other. We assume that the potential part of this operator is non-negative. We add a localized perturbation assuming that it produces two negative isolated eigenvalues being the two lowest spectral values of the resulting perturbed operator. The main result is a lower bound on the gap between these two eigenvalues. It is given explicitly in terms of the geometric properties of the domain and the coefficients of the perturbed operator. We apply this estimate to several asymptotic regimes studying its dependence on various parameters. We discuss specific examples of operators to which the bounds can be applied.
\end{abstract}

Keywords. Spectral gap, lower estimate, second order elliptic operator

Mathematics Subject Classification (2010). Primary 35P15, secondary 35J15

\section{Introduction}

Estimates on eigenvalues of lower bounded self-adjoint Hamilton operators are a classical object of study in mathematical physics and geometry. Among them lower bounds for the distance between successive eigenvalues play an important role. Apart from one-dimensional situations mostly low lying eigenvalues have been studied in the literature. This concerns both Schrödinger operators [6-8, 10,14-16,18] as well as Laplace operators on general Euclidean domains and manifolds $[13,17,19,20]$. While such questions have been considered already in

D. Borisov: Institute of Mathematics of Ufa Scientific Center of RAS, Chernyshevskogo str. 112, 450008 Ufa, Russia; and Faculty of Physics and Mathematics, Bashkir State Pedagogical University, October rev. st. 3a, 450000 Ufa, Russia; borisovdi@yandex.ru

I. Veselić: Fakultät für Mathematik, TU Chemnitz, 09107 Chemnitz, Germany; http://www.tu-chemnitz.de/mathematik/stochastik/ 
the eighties $[7,8,17]$, they are attracting the attention of various authors even in recent time $[10,13,18-20]$.

Note that already the Perron-Frobenius theory gives one the information that the lowest eigenvalue for the operators under consideration cannot be degenerate and thus the distance between the lowest two eigenvalues is positive. This means that lower bounds on this distance are interesting only if one has information on the specific dependence on the parameters of the model under consideration.

The typical result of this genre gives a lower bound on the distance between the lowest and the second lowest eigenvalue in terms of some quantity which is considered as known. This might be the potential of the Schrödinger operator, or more specifically, the distance between two potential wells. In geometric situations one may be interested in the dependence of the gap length in terms of the shape of the underlying domain.

We present a lower bound of the distance between the first and second eigenvalue of a selfadjoint second order differential operator in divergence form on a subdomain of $n$ dimensional Euclidean space. The main features of our result are the following:

- The lower bound is explicit in its dependence on the coefficients of the differential operator, the potential and the geometric data of the domain.

- The explicit estimates allow to deduce interesting results in various asymptotic regimes studied before.

- The result is formulated in terms of a non negative comparison operator and a localized perturbation. The perturbation does not need to be necessarily a potential, but may be itself a differential operator.

- The considered self-adjoint operator and the perturbation are quite general, covering a variety of previously considered as well as new examples.

While the strategy of the proof of our main result is not completely new, we need to develop new tools to deal with the more abstract form of the operator under consideration. This applies in particular for a quantitative version of Harnack's inequality (cf. Section 6) and lower bounds for positivity regions of eigenfunction derivatives (cf. Section 8). One more new ingredient is using Hölder continuity of the eigenfunctions and the estimates for their Hölder norms (cf. Lemma 7.2). It allows us to minimize the restrictions for the smoothness of the eigenfunctions, and therefore, for the coefficients of the studied operators. In addition, all our estimates are explicit, since we are aiming for a quantitative lower bound on the spectral gap in the final result.

In the next section we formulate the main result and discuss the consequences in various asymptotic regimes. It is followed by Section 3 devoted to the discussion of examples which are covered by our general model. Section 4 establishes some preliminary results about the properties of the quadratic forms 
of the operators under consideration. In Section 5 we show that a classical formula for the spectral gap holds for our model. The following section is devoted to a quantitative version of a Harnack inequality. Section 7 deals with pointwise and $L_{p}$-estimates for eigenfunctions, and Section 8 concludes the proof of the main theorem.

\section{Formulation of the problem and the main result}

We introduce the notation used in the paper and formulate the assumptions for our main theorem.

Properties of the quadratic form. Let $n \geqslant 2$ and $\Omega \subseteq \mathbb{R}^{n}$ be a connected open set with $C^{1}$ boundary. Let $0<\nu<\mu<\infty$ and $A_{i j}: \Omega \rightarrow \mathbb{R}, i, j \in$ $\{1, \ldots, n\}$ be bounded functions such that the ellipticity condition

$$
A_{i j}(x)=A_{j i}(x), \quad \nu|\xi|^{2} \leqslant \sum_{i, j=1}^{n} A_{i j}(x) \xi_{i} \xi_{j} \leqslant \mu|\xi|^{2}
$$

holds for all $x=\left(x_{1}, \ldots, x_{n}\right) \in \mathbb{R}^{n}, \xi=\left(\xi_{1}, \ldots, \xi_{n}\right) \in \mathbb{R}^{n}$.

For $V: \Omega \rightarrow \mathbb{R}$ set $V^{+}(x):=\max \{V(x), 0\}, V^{-}(x):=-\min \{V(x), 0\}$. We assume that $V^{-} \in L_{\frac{q}{2}}(\Omega)$ for some $q>n$ and that $V^{+} \in L_{\frac{q}{2}}\left(\Omega^{\prime}\right)$ for each bounded open set $\Omega^{\prime} \subseteq \Omega$. Here we do not exclude the case $\partial \Omega^{\prime} \stackrel{2}{\cap} \partial \Omega \neq \emptyset$.

We introduce the sesquilinear form

$$
\mathfrak{h}[u, v]:=\sum_{i, j=1}^{n}\left(A_{i j} \frac{\partial u}{\partial x_{i}}, \frac{\partial v}{\partial x_{j}}\right)_{L_{2}(\Omega)}+(V u, v)_{L_{2}(\Omega)}
$$

on $L_{2}(\Omega)$ with the domain

$$
\begin{aligned}
\mathcal{D}(\mathfrak{h}) & :=\stackrel{\circ}{2}_{2}^{1}(\Omega, \Gamma) \cap L_{2}\left(\Omega ; V^{+}\right), \\
L_{2}\left(\Omega ; V^{+}\right) & :=\left\{u \in L_{2}(\Omega): \int_{\Omega}|u|^{2}\left(V^{+}+1\right) \mathrm{d} x<\infty\right\},
\end{aligned}
$$

where $\Gamma$ is a (possibly empty) subset of the boundary $\partial \Omega$, and $\stackrel{\circ}{W}_{2}^{1}(\Omega, \Gamma)$ consists of the functions in $W_{2}^{1}(\Omega)$ vanishing on $\Gamma$. This form is symmetric. We will show below (see Lemma 4.1) that it is also lower-semibounded and closed.

The associated selfadjoint operator. By $\mathcal{H}$ we denote the self-adjoint operator associated with the form $\mathfrak{h}$. We observe that this operator has Dirichlet boundary conditions on $\Gamma$ and Neumann ones on the remainder of the boundary.

We denote the spectrum of an selfadjoint operator by the symbol $\sigma(\cdot)$, by $\lambda_{0}:=\inf \sigma(\mathcal{H})$ the infimum of the spectrum of $\mathcal{H}$, and by $\lambda:=\inf \sigma(\mathcal{H}) \backslash\left\{\lambda_{0}\right\}$ the second lowest spectral value. The aim of this paper is to estimate the spectral gap between $\lambda$ and $\lambda_{0}$, in situations where both of these numbers are eigenvalues. 


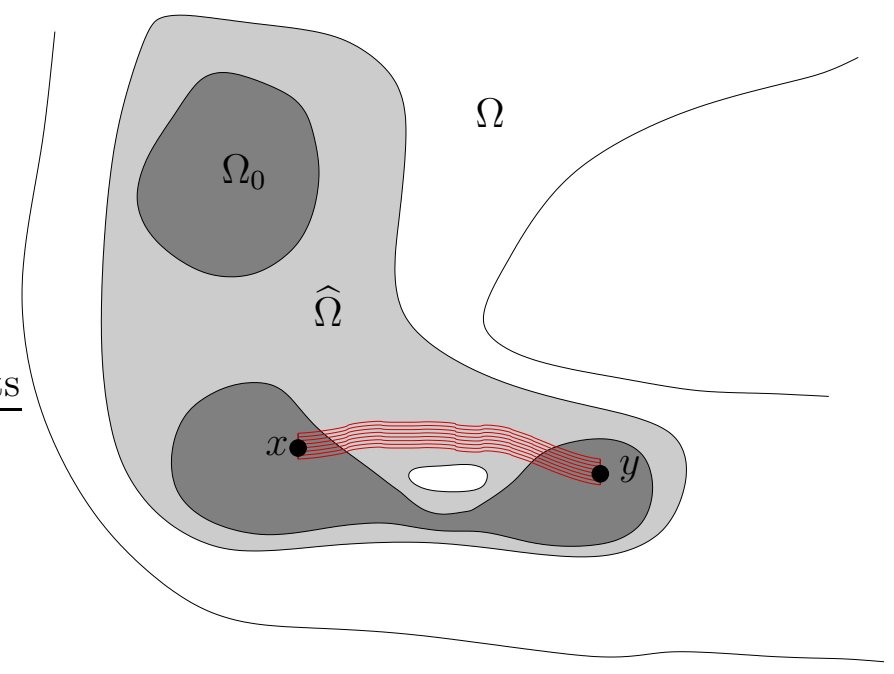

Figure 1: Relation between the sets $\Omega_{0} \subseteq \widehat{\Omega} \subseteq \Omega$ and an admissible (red) cylinder.

Geometric assumptions and the comparison operator. For any $\Omega_{0} \subseteq \Omega$ denote by $\mathcal{H}_{0}$ the self-adjoint operator associated with the same form as in (2), but considered on $L_{2}\left(\Omega \backslash \overline{\Omega_{0}}\right)$ with the domain $\mathscr{W}_{2}^{1}\left(\Omega \backslash \Omega_{0}, \widetilde{\Gamma}\right) \cap L_{2}\left(\Omega \backslash \Omega_{0} ; V^{+}\right)$, where $\widetilde{\Gamma}:=\partial \Omega_{0} \cup \Gamma$. This domain corresponds to the Dirichlet condition on $\widetilde{\Gamma}$.

We assume that there exists open and bounded subsets $\Omega_{0} \subseteq \widehat{\Omega}$ of $\Omega$ such that

- $\operatorname{dist}\left(\Omega_{0}, \partial \Omega\right)=d>0$,

- $\partial \Omega_{0}$ is $C^{1}$-smooth,

- $\lambda_{0}<\lambda<0 \leqslant \inf \sigma\left(\mathcal{H}_{0}\right)$,

- $V^{-} \equiv 0 \quad$ on $\quad \Omega \backslash \Omega_{0}$,

- $\operatorname{dist}\{\widehat{\Omega}, \partial \Omega\} \geqslant \frac{3}{4} d$,

- $\widehat{\Omega}$ is path-connected.

We observe that if $\partial \Omega=\varnothing,(3 \mathrm{a})$ holds true with any $d>0$, and that (3c) excludes the case $V^{-} \equiv 0$, since in this case the operator $\mathcal{H}$ is non-negative.

Admissible cylinders. Given $x, y \in \Omega_{0}$, consider a $C^{2}$-curve connecting these points and lying in $\widehat{\Omega}$. At each point $\hat{x}$ of this curve we consider a $(n-1)$ dimensional disk of radius $r$ having $\hat{x}$ as the center and being orthogonal to the tangential vector of the curve, where $r$ is a small number. As a result, we obtain a curved cylinder or tube along the curve. Since the curve is smooth, 
we can choose $r$ small enough so that the cylinder does not overlap with itself. Since the curve is a closed set it has a positive distance to the boundary of the open set $\widehat{\Omega}$. Thus for $r$ small enough the cylinder corresponding to the curve is a subset of $\widehat{\Omega}$. We call a cylinder with the two mentioned properties admissible.

In Lemma 4.5 we will show that there exist $L, r_{0} \in(0, \infty)$ such that any two points of $\Omega_{0}$ can be connected by an admissible cylinder of length at most $L$ and of radius at least $r_{0}$. The parameter $L$ plays a role of the linear size of the domain $\Omega_{0}$. If $\Omega_{0}$ is a convex domain, then $L$ is the diameter of $\Omega_{0}$, and all admissible cylinders can be chosen straight.

Potential strength parameter. By $\Theta_{n}=\frac{\pi^{\frac{n}{2}}}{\Gamma\left(\frac{n}{2}+1\right)}$ and $\theta_{n}=\frac{2 \pi^{\frac{n}{2}}}{\Gamma\left(\frac{n}{2}\right)}$ we denote the volume of the unit ball and the area of the unit sphere in $\mathbb{R}^{n}$, respectively. Here $\boldsymbol{\Gamma}$ denotes the gamma function. Let $\Omega_{0, t}:=\left\{x: \operatorname{dist}\left(x, \Omega_{0}\right)<t\right\}$, $\widehat{\Omega}_{t}:=\{x: \operatorname{dist}(x, \hat{\Omega})<t\}, B_{r}(a):=\left\{x \in \mathbb{R}^{n}|| x-a \mid<r\right\}$ and

$$
\begin{aligned}
& \hat{q}:=\frac{q}{q-2} \\
& p:= \begin{cases}\frac{n}{n-2}, & \text { for } n>2 \\
\hat{q}+1, & \text { for } n=2\end{cases} \\
& \widehat{V}:=\sup _{a \in \widehat{\Omega}_{\frac{d}{4}}\|V\|_{L_{\frac{q}{2}}\left(B_{\frac{d}{4}}(a)\right)}} \\
&+\Theta_{n}^{\frac{2}{q}}\left(\frac{d}{2}\right)^{\frac{n q}{2}}\left(\frac{8 \nu}{d^{2}}+3^{\frac{n q}{q-n}}\left(\frac{2(p+1)}{\nu}\right)^{\frac{n}{q-n}}\left(\sup _{a \in \Omega_{0}}\left\|V^{-}\right\|_{L_{\frac{q}{2}}\left(B_{\frac{d}{2}}(a)\right)}\right)^{\frac{q}{q-n}}\right) .
\end{aligned}
$$

We observe that $\widehat{V} \neq 0$.

Remark 2.1. In the case $\Gamma=\partial \Omega$, i.e., once one has the Dirichlet condition on the whole boundary $\partial \Omega$ for the operator $\mathcal{H}$, it is possible to replace by zero the term $\frac{8 \nu}{d^{2}}$ in the definition of $\widehat{V}$.

Now we are in the position to formulate our main result. It is a bound for the relative size of the first spectral gap $\frac{\lambda-\lambda_{0}}{|\lambda|}$.

Theorem 2.2. The spectral gap between $\lambda$ and $\lambda_{0}$ obeys the following lower bound

$$
\frac{\lambda-\lambda_{0}}{|\lambda|} \geqslant \frac{\Theta_{n-1} c_{1}^{n-1} \nu}{9 L\left|\Omega_{0, \frac{d}{4}}\right|\left(\left(\frac{p+1}{\nu}\right)^{\frac{n}{q-n}}\left\|V^{-}\right\|_{L_{\frac{q}{2}}\left(\Omega_{0}\right)}^{\frac{q}{q-n}}+4 \mu r_{1}^{-2}\right) c_{2}^{\frac{8 L}{d}}}
$$

where the constants $c_{1}=c_{1}\left(\mu, \nu, n, q, L, d, r_{0}, \widehat{V}\right), c_{2}=c_{2}(\mu, \nu, n, q, d, \widehat{V})$ are 
defined by

$$
\begin{aligned}
& c_{1}=\min \left\{r_{1}\left(3 c_{3} c_{2}^{\frac{8 L}{d}}\right)^{-\frac{1}{\alpha}}, \frac{d}{8}, r_{0}\right\} \\
& c_{2}=2^{\frac{p \hat{q}}{p-\hat{q}}+\frac{p \hat{q}+\hat{q}^{2}}{(p-\hat{q})^{2}}-\frac{2(n-1)}{c_{7}}}\left(\frac{p}{\hat{q}}\right)^{\frac{p^{2} \hat{q}}{c_{7}(p-\hat{q})^{2}}}\left(1+\frac{2 p^{2}}{\hat{q}^{2}}\right)^{\frac{\hat{q}}{2(p-\hat{q})}} \\
& \cdot \max \left\{\left(\Theta_{n} d^{n}\right)^{\frac{2}{c_{7}}},\left(\Theta_{n} d^{n}\right)^{\frac{p+\hat{q}}{c_{7} \hat{q}}}\right\} \max \left\{c_{8}^{\frac{\hat{q}}{2(p-\hat{q})}}, c_{8}^{\frac{\hat{q}^{2}}{2 p(p-\hat{q})}}\right\} \max \left\{c_{9}^{\frac{p \hat{q}}{c_{7}(p-\hat{q})}}, c_{9}^{\frac{p^{2}}{c_{7}(p-\hat{q})}}\right\} \\
& r_{1}=\min \left\{\Theta_{n}^{-\frac{1}{n}}\left(\frac{\nu}{12(p+1)^{2} \widehat{V}}\right)^{\frac{q}{2(q-n)}}, \frac{d}{4}\right\}, \\
& \alpha=\min \left\{-\log _{4}\left(1-2^{-c_{4}}\right), 1-\frac{n}{q}\right\} \text {, } \\
& c_{3}=4^{\alpha} \max \left\{2, \frac{2^{c_{4}+2} \nu}{9 \sqrt{6} \mu(p+1) \Theta_{n}^{\frac{1}{n}}}\right\} \text {, } \\
& c_{4}=3+81 \cdot 2^{n+9}\left(\theta_{n}+1\right)^{2} n^{-2} \mu^{2} \nu^{-2} c_{5}^{\frac{2(n-1)}{n}}, \\
& c_{5}=\max \left\{2^{2 n+1} \Theta_{n}^{-1}, 4^{\frac{q^{2} n^{2}}{(q-n)^{2}}} c_{6}^{\frac{q n}{q-n}}\right\} \text {, } \\
& c_{6}=9 \cdot 2^{2 n+9} \Theta_{n}^{\frac{1-q}{q}} n^{-1}\left(\theta_{n}+1\right) \mu \nu^{-1} \text {, } \\
& c_{7}=\frac{\Theta_{n}^{\frac{1}{2}} \nu^{\frac{1}{2}}}{2^{n+1} e C_{9}}\left(2^{n+4} \mu \Theta_{n}+2^{n\left(1+\frac{2}{q}\right)-3} \widehat{V} \Theta_{n}^{\frac{1}{\hat{q}}} d^{2\left(1-\frac{n}{q}\right)}\right)^{-\frac{1}{2}}, \\
& c_{8}=\frac{(p+1)^{4}}{p}\left(2^{11}\left(1+\frac{4 \mu}{\nu}\right) \frac{\Theta_{n}^{\frac{2}{q}} d^{\frac{2 n}{q}-2}}{4^{\frac{2 n}{q}}}+4 \frac{\widehat{V}}{\nu}\right)\left(2^{11}\left(1+\frac{\mu}{\nu}\right) \frac{\Theta_{n}^{\frac{2}{q}} d^{\frac{2 n}{q}-2}}{4^{\frac{2 n}{q}}}+\frac{\widehat{V}}{\nu}\right), \\
& c_{9}=\frac{4(p+1)^{2} p \hat{q} \widehat{V}}{(p-\hat{q})^{2} \nu}\left(1+\frac{c_{7}}{\hat{q}}\right)+2^{11-\frac{4 n}{q}}(p+1)^{2} \hat{q} \Theta_{n}^{\frac{2}{q}} d^{\frac{2 n}{q}-2} \\
& \cdot\left(\frac{1}{p}+\frac{4 p}{(p-\hat{q})^{2}} \frac{\mu}{\nu}\right) \log _{\frac{p}{\tilde{q}}}^{2} \frac{2 p^{3}}{c_{7} \hat{q}^{2}} \text {. }
\end{aligned}
$$

Remark 2.3. 1 . Note that $|\lambda|$ is the distance of $\lambda$ to zero. Hence, the distance between $\lambda$ and $\lambda_{0}$ can not be much smaller than that from $\lambda$ to zero. In particular, it means that $\lambda_{0}$ can not be an accumulation point for the eigenvalues of $\mathcal{H}$.

2. Let us note that if there exists a further eigenvalue $\widetilde{\lambda} \in(\lambda, 0)$ we could give a lower bound similar to (5) for the distance $\tilde{\lambda}-\lambda_{0}$. It turns out however, that the trivial comparison $\tilde{\lambda}-\lambda_{0} \geqslant \lambda-\lambda_{0}$ gives us a better estimate. 
3. The estimate (5) is invariant under the multiplication of the operator $\mathcal{H}$ by a constant.

One of the main advantages of Theorem 2.2 is that the size of the lower bound is given explicitly. Although the formulae look quite bulky, it is possible to study effectively the dependence of the right hand side in (5) w.r.t. to various parameters. This is demonstrated in the next theorems.

Theorem 2.4. For $L$ large enough the spectral gap between $\lambda$ and $\lambda_{0}$ satisfies the estimate

$$
\frac{\lambda-\lambda_{0}}{|\lambda|} \geqslant c_{10} L^{-n-1} e^{-c_{11} L}
$$

where $c_{10}>0$ depends on $\mu, \nu, n, q, d, \widehat{V},\left\|V^{-}\right\|_{L_{\frac{q}{2}}(\Omega)}$, and $c_{11}>0$ depends on $\mu, \nu, n, q, d, \widehat{V}$.

Here the phrase "for $L$ large enough" should be understood as: if we keep all parameters except $L$ fixed, there exists some $L_{0}$, depending on these other parameters, such that for $L \geqslant L_{0}$ the claimed estimate holds true.

As it was said above, the parameter $L$ characterizes the linear size of the domain $\Omega_{0}$. Theorem 2.5 shows how our estimate depends on $L$; it turns out that the dependence is very simple. We also observe that the estimate is exponentially small as $L \rightarrow+\infty$.

Theorem 2.5. For $\widehat{V}$ small enough the spectral gap between $\lambda$ and $\lambda_{0}$ satisfies the estimate

$$
\frac{\lambda-\lambda_{0}}{|\lambda|} \geqslant c_{12} L^{-n-1} e^{-c_{13} L},
$$

where $c_{12}>0$ depends on $n, q, \mu, \nu, d, r_{0}$, and $c_{13}>0$ depends on $n, q, \mu, \nu, d$.

This theorem addresses the case of a small potential, which can be considered as a weak coupling regime. As it is well known, in this case the eigenvalues, if they exist, are close to the threshold of the unperturbed spectrum, in our case, to zero. This fact is reflected by the estimate (7), since $|\lambda|$ tends to zero as $\widehat{V} \rightarrow+0$.

Theorem 2.6. For $\nu$ small enough and $\frac{\mu}{\nu}=$ constant, the spectral gap between $\lambda$ and $\lambda_{0}$ satisfies the estimate

$$
\frac{\lambda-\lambda_{0}}{|\lambda|} \geqslant c_{14} L^{-n-1}\left(c_{15} \nu\right)^{c_{16} \frac{L}{\sqrt{\nu}}}
$$

where $c_{14}>0$ depends on $d, n, q, c_{15}>0$ depends on $d, n, q, \widehat{V}, \frac{\mu}{\nu}$, and $c_{16}>0$ depends on $d, n, q, \frac{\mu}{\nu}$. 
This theorem treats the semiclassical regime. In this regime the functions $A_{i j}$ read as follows $A_{i j}=\hbar^{2} \widetilde{A}_{i j}$, where $\hbar \rightarrow+0$. Hence, both the parameters $\mu$ and $\nu$ tend to zero, while $\frac{\mu}{\nu}$ remains constant. It is known that in certain semiclassical situations the distance between the first two eigenvalues is exponentially small with respect to $\hbar \approx \sqrt{\nu}$, see e.g., [14]. Theorem 2.6 gives a lower bound which decreases slightly faster than exponentially w.r.t. $\sqrt{\nu} \rightarrow 0$.

Theorem 2.7. For $\nu$ small enough and $\mu=$ constant, the spectral gap between $\lambda$ and $\lambda_{0}$ satisfies the estimate

$$
\frac{\lambda-\lambda_{0}}{|\lambda|} \geqslant c_{18} L^{-n-1} \nu^{1+\frac{q(n+1)}{2(q-n)}} \exp \left(-c_{19} c_{20} \nu^{-\frac{2(n-1) q}{q-n}}\right)\left(c_{21} \nu^{-1} \log _{\frac{p}{\tilde{q}}} \nu\right)^{-c_{22} c_{20} \frac{L}{\sqrt{\nu}}}
$$

where $c_{20}=\exp \left(c_{23} \nu^{-\frac{2(n-1) q}{q-n}}\right), c_{18}=c_{18}(n, d, \mu, \widehat{V})>0, c_{i}=c_{i}(n, q, \mu)>0$ for $i=19,23$, and $c_{i}=c_{i}(n, q, d, \mu, \widehat{V})>0$ for $i=21,22$.

Theorem 2.7 is adapted to the models of the photonic crystals, see Example 3 in Section 3.

\section{Applications}

In this section we give a series of the examples illustrating possible applications of our results.

3.1. Second order differential operator with localized perturbation. Let $A_{i j}^{(0)}=A_{i j}^{(0)}(x)$ be bounded real-valued functions defined on $\Omega$ such that

$$
A_{i j}^{(0)}(x)=A_{j i}^{(0)}(x), \quad \nu_{0}|\xi|^{2} \leqslant \sum_{i, j=1}^{n} A_{i j}^{(0)}(x) \xi_{i} \xi_{j} \leqslant \mu_{0}|\xi|^{2}, \quad x \in \Omega
$$

where $0<\nu_{0}<\mu_{0}$ are constants. By $V^{(0)}$ we denote a non-negative function defined on $\Omega$, so that $V^{(0)} \in L_{\frac{q}{2}}\left(\Omega^{\prime}\right)$ for each bounded domain $\Omega^{\prime} \subseteq \bar{\Omega}$. We introduce the self-adjoint operator $\mathcal{H}^{(0)}$ associated with the form

$$
\mathfrak{h}^{(0)}[u, v]:=\sum_{i, j=1}^{n}\left(A_{i, j}^{(0)} \frac{\partial u}{\partial x_{i}}, \frac{\partial v}{\partial x_{j}}\right)_{L_{2}(\Omega)}+\left(V^{(0)} u, v\right)_{L_{2}(\Omega)}
$$

on $L_{2}(\Omega)$ with the domain $\stackrel{\circ}{W}_{2}^{1}(\Omega, \Gamma) \cap L_{2}\left(\Omega ; V^{+}\right)$, where $\Gamma$ has the same meaning as above. Let $\Omega_{0}$ be a bounded subdomain of $\Omega$ with $C^{1}$-boundary and separated from $\partial \Omega$ by a positive distance, and $A_{i j}^{(1)}=A_{i j}^{(1)}(x), V^{(1)}=V^{(1)}(x)$ be bounded real-valued functions defined on $\Omega$ with supports in $\Omega_{0}$, and such that the functions $A_{i j}:=A_{i j}^{(0)}+A_{i j}^{(1)}, V:=V^{(0)}+V^{(1)}$ satisfy the conditions (1), 
$V^{-} \in L_{\frac{q}{2}}(\Omega)$ for some $q>n$, and $V^{+} \in L_{\frac{q}{2}}\left(\Omega^{\prime}\right)$ for each bounded domain $\Omega^{\prime} \subseteq \bar{\Omega}$. Then we can consider the operator $\mathcal{H}$ defined via the coefficients $A_{i j}$ and $V$.

We also suppose that $\lambda_{0}=\inf \sigma(\mathcal{H})<0$ and $\lambda<0$ are two isolated eigenvalues of $\mathcal{H}$. Then the domain $\Omega_{0}$ satisfies the assumption (3). Indeed, in this case the operator $\mathcal{H}_{0}$ is determined only by $A_{i j}^{(0)}$ and $V^{(0)}$ and is independent of $A_{i j}^{(1)}$, $V^{(1)}$. It remains to suppose that the domain $\Omega_{0}$ satisfies the assumptions $(3 \mathrm{e})$ and (3f), and then one can apply Theorems $2.2-2.7$ to the operator $\mathcal{H}$.

A particular choice of the domain $\Omega$ would be a waveguide-type domain, as depicted in Figure 2. It allows us to apply our results to such domains which are of interest in the physical theory of quantum waveguides. One more possible choice is a perforated domain with a perturbation localized on a bounded subdomain, cf. Figure 3.

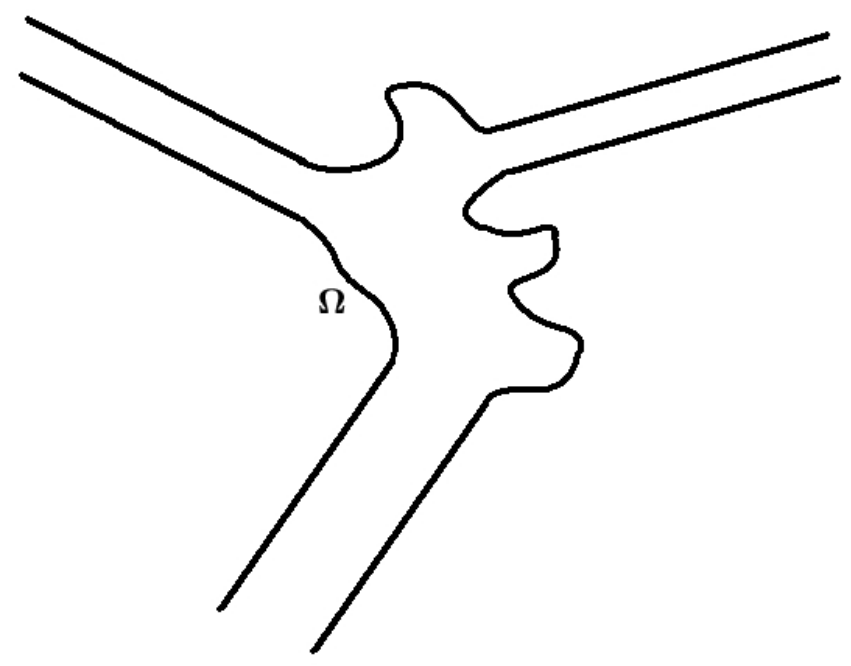

Figure 2: Waveguide

3.2. Perturbation by a singular surface measure. Although the results of the paper are formulated only for usual differential operators, they can be applied to a more general class of operators. Namely, if some operator can be transformed by a unitary transformation to the above considered differential operator, one can apply our results to such operators as well. An example for such an operator would be the negative Laplacian plus a singular potential which equals the Hausdorff measure supported on a manifold of codimension one. Such operators can be transformed to the above considered differential operators, see Example 5 in [1]. 
3.3. Photonic crystals. Photonic crystals are periodic dielectric media with the property that the electromagnetic waves with certain frequencies cannot propagate in it. To achieve this property one uses high contrast materials. An instance would be the case where the dielectric constant of the material takes on two positive values whose quotient is very large. We refer for more details to the review [11] and the references therein.

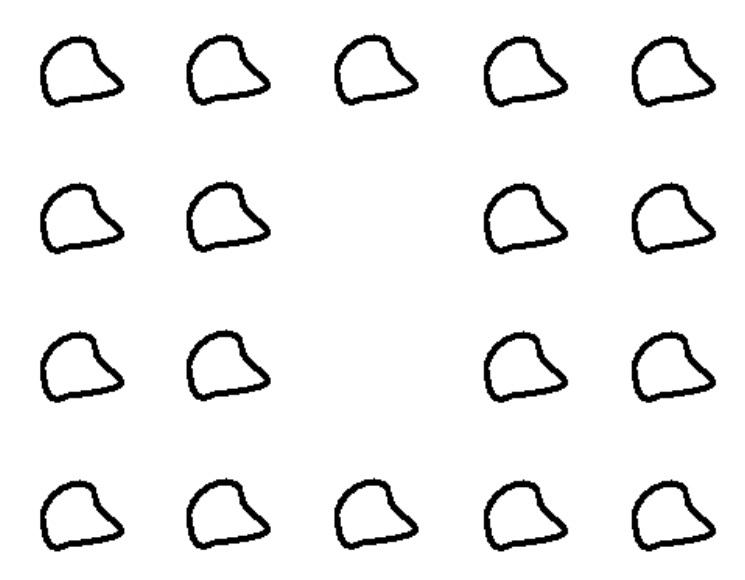

Figure 3: Perforated domain

Mathematical models which describe photonic crystals are elliptic differential operators. The high contrast properties of the medium are described by the coefficients of the differental operator. In terms of our notation these properties can be formulated as follows. The set $\Omega$ equals $\mathbb{R}^{n}$, the coefficients $A_{i j}$ and $V$ are introduced in the same way as in Example 1. The functions $A_{i j}^{(0)}$ are bounded, periodic with respect to the lattice $\mathbb{Z}^{n}$, and each of them takes on only two values $0<\nu_{i j} \ll \mu_{i j}<\infty$.

The potential $V^{(0)}$ is $\mathbb{Z}^{n}$-periodic as well. The coefficients $A_{i j}^{(1)}, V^{(1)}$ of the perturbation are bounded, compactly supported functions. In this case we think of $\mu$ as a fixed positive real, while $\nu$ is positive, but very close to zero. Hence, one can apply in this case Theorem 2.2 , or Theorem 2.7 .

3.4. Distant perturbations. Distant perturbations are perturbations which are localized on a finite number of bounded domains with large distances between them. More precisely, let $\Omega=\mathbb{R}^{n}$, and let $Q_{k} \subset \mathbb{R}^{n}$ be a finite number of bounded domains such that the distance between $Q_{k}$ and $Q_{m}$ for $k \neq m$ is at least $l>0$. Define the operator $\mathcal{H}^{(0)}$ and its coefficients $A_{i j}^{(0)}, V^{(0)}$ as in Example 1. Assume that the coefficients $A_{i j}^{(1)}, V^{(1)}$ are supported in $\bigcup_{k} Q_{k}$. The resulting differential operator $\mathcal{H}$ describes a model for distant or separated perturbations. Such models were formulated and studied in their most general 
form in the references [1] and [2]. There one can also find a review of earlier results.

Our theorems apply to such problems, too. For $\Omega_{0}$ one chooses the union $\bigcup_{k} Q_{k}$. Then the main feature of $\Omega_{0}$ is that the parameter $L>l$ is large, since $l$ is assumed large. In this case Theorem 2.4 says that the spectral gaps are bounded from below by an exponentially small quantity w.r.t. L. Such situations have been studied in previous literature, see for instance, $[1,2,6,7,10]$. Let us discuss the results of the two most recent papers in more detail. The main results of the $[1,2]$ are asymptotic expansions for the eigenvalues of the operators with distant perturbations. These asymptotics imply that the spectral gap we consider here is exponentially small w.r.t. to $L$. In this respect the result of Theorem 2.4 is in a good agreement with that of $[1,2]$. However, in general the constants in the estimate in Theorem 2.4 are not optimal. In this respect the results in $[1,2]$ are better than Theorem 2.4.

\section{Preliminaries}

In this section we prove that the operator $\mathcal{H}$ is well-defined and we study certain properties of its ground state. We also prove the existence of bounds $L$ and $r_{0}$ for the admissible cylinders connecting the points in $\Omega_{0}$.

In the space $\mathcal{D}(\mathfrak{h})$ we introduce the scalar product given by

$$
(u, v)_{\mathcal{D}(\mathfrak{h})}=(u, v)_{W_{2}^{1}(\Omega)}+(u, v)_{L_{2}\left(\Omega ; V^{+}\right)}, \quad(u, v)_{L_{2}\left(\Omega ; V^{+}\right)}:=\int_{\Omega}\left(1+V^{+}\right) u v \mathrm{~d} x
$$

¿From [9, Chapter 10, Section 37.2, Theorem 5'] it follows that $\mathcal{D}(\mathfrak{h})$ equipped with the scalar product forms a Hilbert space.

Lemma 4.1. The form $\mathfrak{h}$ is lower semibounded and closed. The inequality

$$
\begin{aligned}
\mathfrak{h}[u, u] & \geqslant-C_{1}\|u\|_{L_{2}(\Omega)}^{2}, \\
C_{1} & :=\frac{8 \nu}{d^{2}}+3^{\frac{n q}{q-n}}\left(\frac{2(p+1)}{\nu}\right)^{\frac{n}{q-n}}\left(\sup _{a \in \Omega_{0}}\left\|V^{-}\right\|_{L_{\frac{q}{2}}\left(B_{\frac{d}{2}}(a)\right)}\right)^{\frac{q}{q-n}},
\end{aligned}
$$

holds true.

Proof. Let us prove first that the form is lower semibounded and closed. Given a point $a \in \Omega_{0}$, consider a ball $B_{\frac{d}{2}}(a)$. Let $\zeta=\zeta(t)$ be a function equalling one as $t \leqslant \frac{d}{2}$ and $2-2 t / d$ as $t>\frac{d}{2}$. Then for any function $u \in \mathcal{D}(\mathfrak{h})$ we have $\zeta(|\cdot-a|) u \in \stackrel{\circ}{W}_{2}^{1}\left(B_{d}(a)\right)$. It follows from the definition of $V^{-}$, the Hölder and 
Young inequalities, and [12, Chapter II, Section 2, Inequality (2.11)] that

$$
\begin{aligned}
& \left|\left(V^{-} u, u\right)_{L_{2}\left(B_{\frac{d}{2}}(a)\right)}\right| \\
& \leqslant\left\|V^{-}\right\|_{L_{\frac{q}{2}}\left(B_{\frac{d}{2}}(a)\right)}\|u\|_{L_{\frac{2 q}{q-2}}^{2}\left(B_{\frac{d}{2}}(a)\right)} \\
& \leqslant(p+1)^{\frac{n}{q}}\left\|V^{-}\right\|_{L_{\frac{q}{2}}\left(B_{\frac{d}{2}}(a)\right)}\|\nabla \zeta u\|_{L_{2}\left(B_{d}(a)\right)}^{\frac{2 n}{q}}\|\zeta u\|_{B_{d}(a)}^{2\left(1-\frac{n}{q}\right)} \\
& \leqslant \frac{(p+1)^{\frac{n}{q}}}{q}\left\|V^{-}\right\|_{L_{\frac{q}{2}}\left(B_{\frac{d}{2}}(a)\right)}\left(n \varepsilon\|\nabla \zeta u\|_{L_{2}\left(B_{d}(a)\right)}^{2}+(q-n) \varepsilon^{-\frac{n}{q-n}}\|u\|_{L_{2}\left(B_{d}(a)\right)}^{2}\right) \\
& \leqslant \frac{(p+1)^{\frac{n}{q}}}{q}\left\|V^{-}\right\|_{L_{\frac{q}{2}}\left(B_{\frac{d}{2}}(a)\right)} \\
& \cdot\left(2 n \varepsilon\|\nabla u\|_{L_{2}\left(B_{d}(a)\right)}^{2}+\left((q-n) \varepsilon^{-\frac{n}{q-n}}+8 n \varepsilon d^{-2}\right)\|u\|_{L_{2}\left(B_{d}(a)\right)}^{2}\right),
\end{aligned}
$$

where $\zeta=\zeta(|\cdot-a|)$, and $\varepsilon>0$ is arbitrary. It is clear that the set $\Omega_{0}$ can be covered by a finite set of balls $B_{\frac{d}{2}}(a)$ with $a \in \Omega_{0}$, such that each point of $\Omega_{0}$ belongs at most to $3^{n}$ such balls. Then by the last estimate we have

$$
\begin{aligned}
\left|\left(V^{-} u, u\right)_{L_{2}\left(\Omega_{0}\right)}\right|= & \sum_{a}\left|\left(V^{-} u, u\right)_{L_{2}\left(B_{\frac{d}{2}}(a)\right)}\right| \\
\leqslant & 3^{n} \frac{(p+1)^{\frac{n}{q}}}{q} \sup _{a \in \Omega_{0}}\left\|V^{-}\right\|_{L_{\frac{q}{2}}\left(B_{\frac{d}{2}}(a)\right)} \\
& \cdot\left(2 n \varepsilon\|\nabla u\|_{L_{2}(\Omega}^{2}+\left((q-n) \varepsilon^{-\frac{n}{q-n}}+8 n \varepsilon d^{-2}\right)\|u\|_{L_{2}\left(B_{d}(a)\right)}^{2}\right) .
\end{aligned}
$$

We choose

$$
\varepsilon:=\frac{q \nu}{2 \cdot 3^{n} n(p+1)^{\frac{n}{q}} \sup _{a \in \Omega_{0}}\left\|V^{-}\right\|_{L_{\frac{q}{2}}\left(B_{\frac{d}{2}}(a)\right)}}
$$

and obtain

$$
\begin{gathered}
\left|\left(V^{-} u, u\right)_{L_{2}(\Omega)}\right| \leqslant \nu\|\nabla u\|_{L_{2}\left(\Omega_{0}\right)}^{2}+\varepsilon_{1}\|u\|_{L_{2}\left(\Omega_{0}\right)}^{2}, \\
\varepsilon_{1}:=\frac{8 \nu}{d^{2}}+3^{\frac{n q}{q-n}}\left(1-\frac{n}{q}\right)\left(\frac{2 n}{q \nu}\right)^{\frac{n}{q-n}}(p+1)^{\frac{n}{q-n}}\left(\sup _{a \in \Omega_{0}}\left\|V^{-}\right\|_{L_{\frac{q}{2}}\left(B_{\frac{d}{2}}(a)\right)}\right)^{\frac{q}{q-n}} .
\end{gathered}
$$

This inequality and the definition of the form $\mathfrak{h}$ imply that this form is lowersemibounded. Substituting the obtained inequalities into the definition of $\mathfrak{h}$, taking into account $(3 \mathrm{~d})$, and applying the estimate $t^{\frac{t}{1-t}}(1-t) \leqslant 1, t \in[0,1]$ with $t=\frac{n}{q}$, we arrive at (10). It also follows from (11) with

$$
\varepsilon:=\frac{q \nu}{4 \cdot 3^{n} n(p+1)^{\frac{n}{q}} \sup _{a \in \Omega_{0}}\left\|V^{-}\right\|_{L_{\frac{q}{2}}\left(B_{\frac{d}{2}}(a)\right)}}
$$

that $\frac{\nu}{2}\|\nabla u\|_{L_{2}(\Omega)}^{2}-C\|u\|_{L_{2}(\Omega)}^{2}+\|u\|_{L_{2}\left(\Omega ; V^{+}\right)}^{2} \leqslant \mathfrak{h}(u, u) \leqslant \mu\|\nabla\|_{L_{2}(\Omega)}^{2}+\|u\|_{L_{2}\left(\Omega ; V^{+}\right)}^{2}$. Employing these inequalities, one can check by the definition that the form $\mathfrak{h}$ is closed. 
Remark 4.2. In the case of the Dirichlet condition on $\partial \Omega$ for the operator $\mathcal{H}$ $(\Gamma=\partial \Omega)$ the term $\frac{8 \nu}{d^{2}}$ in the definition of $C_{1}$ can be replaced by zero. Indeed, it appeared just due to the using of the cut-off function $\zeta$ in the proof. The presence of this function gave the possibility of applying the results of $[12$, Chapter II, Section 2, Inequality (2.11)]. Once we have the Dirichlet condition on $\partial \Omega$, it is possible to these results directly to the function $u$ without using $\zeta$.

Lemma 4.3. There exists an eigenfunction $\psi_{0}$ of $\mathcal{H}$ associated with $\lambda_{0}$ such that $\psi_{0}(x)>0$ for all $x \in \Omega$. For any compact $\Omega^{\prime} \subset \Omega$ there exist constants $C, \tilde{C} \in(0, \infty)$ such that

$$
C \leq \inf _{\Omega^{\prime}} \psi_{0} \leq \sup _{\Omega^{\prime}} \psi_{0} \leq \tilde{C} .
$$

Proof. By the assumption, $\lambda_{0}$ is an eigenvalue and consequently there exists a $\psi_{0} \in \mathcal{D}(\mathcal{H})$ with $\mathcal{H} \psi_{0}=\lambda_{0} \psi_{0}$. Then $\left|\psi_{0}\right|$ is still in the domain of $\mathfrak{h}$ and is a weak solution of

$$
\mathcal{H}\left|\psi_{0}\right|=\lambda_{0}\left|\psi_{0}\right|
$$

since $\mathfrak{h}\left[\left|\psi_{0}\right|,\left|\psi_{0}\right|\right]=\mathfrak{h}\left[\psi_{0}, \psi_{0}\right]=\lambda_{0}$. Moreover, $\lambda_{0}\left|\psi_{0}\right|<0$. In view of equation (12) we can apply the Harnack inequality to $\left|\psi_{0}\right|$ (see [5, Section 8.8]), which shows that on any $\Omega^{\prime} \Subset \Omega$ there is are uniform positive lower and upper bounds on $\psi_{0}$.

Lemma 4.4. The set of the functions in $C^{\infty}(\Omega)$ vanishing in a neighbourhood of $\Gamma$ is dense in $\mathcal{D}(\mathfrak{h})$ in the topology induced by $(\cdot, \cdot)_{\mathcal{D}(\mathfrak{h})}$.

This lemma follows from Theorems 1.8.1 and 1.8.2 in [3].

Lemma 4.5. There exists two positive numbers $L$ and $r_{0}$ such that any two points in $\bar{\Omega}_{0}$ can be connected by an admissible cylinder of the length at most $L$ and of the radius at least $r_{0}$.

Proof. We fix a point $y \in \Omega_{0}$ and introduce the sets $\Xi_{m, k}, m, k \in \mathbb{N}$, consisting of all $x \in \widehat{\Omega}$ such that $x$ can be connected with $y$ by an admissible cylinder of the length less than $m$ and the radius less than $\frac{1}{k}$. One can see easily that $\Xi_{m, k}$ are open sets, and $\Xi_{m, k} \subset \Xi_{\tilde{m}, \tilde{k}}$ if $m<\widetilde{m}, k<\widetilde{k}$. By the assumption (3e) we conclude that for each $x \in \widehat{\Omega}$ there exists a curve connecting $x$ and $y$ lying in the open set $\hat{\Omega}$. Due to (3f) it is possible to chose a sufficiently small radius such that the corresponding cylinder connecting $x$ and $y$ is admissible. Therefore, $\bar{\Omega}_{0} \subseteq \bigcup_{m, k} \Xi_{m, k}$. The set $\bar{\Omega}_{0}$ being compact, we conclude that there exists a finite cover of $\bar{\Omega}_{0}$ by the sets $\Xi_{m, n}$. In view of monotonicity of these sets w.r.t. $m, n$ it implies that there exists $K$ such that $\bar{\Omega}_{0} \subset \Xi_{K, K}$. Hence, each point in $\Omega_{0}$ can be connected with $y$ by an admissible cylinder of the length at most $K$ 
and of the radius at least $\frac{1}{K}$.

Let us prove that any two points $x_{1}, x_{2} \in \Omega_{0}$ can be connected by an admissible cylinder of the length at most $3 K$ and the radius at least $\frac{1}{4 K}$. It is true, if they can connected by an admissible cylinder of the length at most $K$ and the radius at least $\frac{1}{K}$. If not, we connect them with $y$ by admissible cylinder of the length at most $K$ and of the radius at least $\frac{1}{K}$. As a result, we have a cylinder connecting $x_{1}, x_{2}$, having the length $2 K$ and the radius $\frac{1}{K}$. Denote this cylinder by $T$. The corresponding curve connecting $x_{1}$ and $x_{2}$ is piecewise $C^{2}$-smooth, with possible non-smoothness at $y$. It is clear that we can replace by a $C^{2}$-smooth curve of the length at most $3 K$ so that there exists an admissible cylinder corresponding to this curve, having the radius $\frac{1}{4 K}$ and lying inside $T$.

\section{A formula for spectral gaps}

In this section we use a ground state transformation to establish a formula for the lowest spectral gap. For this purpose we will need the next lemma which follows directly from [12, Chapter III, Section 13, Theorem 13.1] and [12, Chapter III, Section 14, Theorem 14.1].

Lemma 5.1. Let $\lambda$ be an eigenvalue of $\mathcal{H}$. Then every eigenfunction $\psi$ associated to $\lambda$ is continuous in $\Omega$ up to the boundary.

Our next aim is to derive a formula for expressions of the form $\mathfrak{h}\left[u \psi_{0}, u \psi_{0}\right]$, where $\psi_{0}$ denotes as before the ground state. Let $u \in C_{0}^{\infty}(\bar{\Omega})$. One can easily check that $u \psi_{0} \in \mathcal{D}(\mathfrak{h}), u^{2} \psi_{0} \in \mathcal{D}(\mathfrak{h})$. Hence, $\mathfrak{h}\left[u \psi_{0}, u \psi_{0}\right]$ is well-defined. Taking into account the symmetry of $A_{i j}$ (see (1)) and the definition of $\psi_{0}$, we check by direct calculation $\sum_{i, j=1}^{n}\left(A_{i, j} \frac{\partial u \psi_{0}}{\partial x_{i}}, \frac{\partial u \psi_{0}}{\partial x_{j}}\right)_{L_{2}(\Omega)}=\sum_{i, j=1}^{n}\left(A_{i, j} \psi_{0} \frac{\partial u}{\partial x_{i}}, \psi_{0} \frac{\partial u}{\partial x_{j}}\right)_{L_{2}(\Omega)}$ $+\sum_{i, j=1}^{n}\left(\left(A_{i, j} \psi_{0} \frac{\partial u}{\partial x_{i}}, u \frac{\partial \psi_{0}}{\partial x_{j}}\right)_{L_{2}(\Omega)}+\left(A_{i, j} u \frac{\partial \psi_{0}}{\partial x_{i}}, \frac{\partial u \psi_{0}}{\partial x_{j}}\right)_{L_{2}(\Omega)}\right)$ and

$$
\begin{aligned}
& \sum_{i, j=1}^{n}\left(\left(A_{i, j} \psi_{0} \frac{\partial u}{\partial x_{i}}, u \frac{\partial \psi_{0}}{\partial x_{j}}\right)_{L_{2}(\Omega)}+\left(A_{i, j} u \frac{\partial \psi_{0}}{\partial x_{i}}, \frac{\partial u \psi_{0}}{\partial x_{j}}\right)_{L_{2}(\Omega)}\right) \\
& =\sum_{i, j=1}^{n}\left(\left(A_{i, j} \frac{\partial \psi_{0}}{\partial x_{i}}, u \psi_{0} \frac{\partial u}{\partial x_{j}}\right)_{L_{2}(\Omega)}+\left(A_{i, j} \frac{\partial \psi_{0}}{\partial x_{i}}, u \frac{\partial u \psi_{0}}{\partial x_{j}}\right)_{L_{2}(\Omega)}\right) \\
& =\sum_{i, j=1}^{n}\left(A_{i, j} \frac{\partial \psi_{0}}{\partial x_{i}}, \frac{\partial u^{2} \psi_{0}}{\partial x_{j}}\right)_{L_{2}(\Omega)} \\
& =\lambda_{0}\left\|u \psi_{0}\right\|_{L_{2}(\Omega)}^{2}-\left(V \psi_{0}, u^{2} \psi_{0}\right)_{L_{2}(\Omega)} .
\end{aligned}
$$


We substitute these identities into the definition of $\mathfrak{h}$ to obtain

$$
\mathfrak{h}\left[u \psi_{0}, u \psi_{0}\right]=\sum_{i, j=1}^{n}\left(A_{i, j} \psi_{0} \frac{\partial u}{\partial x_{i}}, \psi_{0} \frac{\partial u}{\partial x_{j}}\right)_{L_{2}(\Omega)}+\lambda_{0}\left\|u \psi_{0}\right\|_{L_{2}(\Omega)}^{2}
$$

Let $\psi$ be an eigenfunction associated to the eigenvalue $\lambda$. Let $\Omega^{\prime}$ be an arbitrary bounded subdomain of $\Omega$ separated from $\partial \Omega$ by a positive distance. The last relation and (1) imply

$$
\begin{aligned}
\frac{\mathfrak{h}\left[u \psi_{0}, u \psi_{0}\right]-\lambda_{0}\left\|u \psi_{0}\right\|_{L_{2}(\Omega)}^{2}}{\|\psi\|_{L_{2}(\Omega)}^{2}} & \geqslant \frac{1}{\|\psi\|_{L_{2}(\Omega)}^{2}} \sum_{i, j=1}^{n}\left(A_{i, j} \psi_{0} \frac{\partial u}{\partial x_{i}}, \psi_{0} \frac{\partial u}{\partial x_{j}}\right)_{L_{2}\left(\Omega^{\prime}\right)} \\
& \geqslant \frac{\nu\left\|\psi_{0} \nabla u\right\|_{L_{2}\left(\Omega^{\prime}\right)}^{2}}{\|\psi\|_{L_{2}(\Omega)}^{2}}
\end{aligned}
$$

We would like to apply the last formula to $u=\frac{\psi}{\psi_{0}}$. However, since we do not know whether $\frac{\psi}{\psi_{0}}$ is in $u \in C_{0}^{\infty}(\bar{\Omega})$, we use an approximation argument. Let $v_{m} \in C_{0}^{\infty}(\Omega)$ be a sequence approximating $\psi$ in $\mathcal{D}(\mathfrak{h})$. Such a sequence exists by Lemma 4.4. We take $u=u_{m}:=\frac{v_{m}}{\psi_{0}}$ and pass to the limit as $m \rightarrow+\infty$ in (13). Then the left hand side converges to $\frac{\mathfrak{h}[\psi, \psi]-\lambda_{0}\|\psi\|_{L_{2}(\Omega)}^{2}}{\|\psi\|_{L_{2}(\Omega)}^{2}}=\lambda-\lambda_{0}$. Using Lemma 4.3 and Lemma 4.4 we see that the functions $u_{m}$ converge to $\frac{\psi}{\psi_{0}}$ in $W_{2}^{1}\left(\Omega^{\prime}\right)$. Therefore,

$$
\lambda-\lambda_{0} \geqslant \frac{\nu}{\|\psi\|_{L_{2}(\Omega)}^{2}}\left\|\psi_{0} \nabla \frac{\psi}{\psi_{0}}\right\|_{L_{2}\left(\Omega^{\prime}\right)}^{2} \geqslant \frac{\nu \inf _{\Omega^{\prime}} \psi_{0}^{2}}{\|\psi\|_{L_{2}(\Omega)}^{2}}\left\|\nabla \frac{\psi}{\psi_{0}}\right\|_{L_{2}\left(\Omega^{\prime}\right)}^{2} .
$$

It follows from the Cauchy-Schwarz inequality that $\left\|\nabla \frac{\psi}{\psi_{0}}\right\|_{L_{1}\left(\Omega^{\prime}\right)}^{2} \leqslant\left|\Omega^{\prime}\right|\left\|\nabla \frac{\psi}{\psi_{0}}\right\|_{L_{2}\left(\Omega^{\prime}\right)}^{2}$. Hence,

$$
\lambda-\lambda_{0} \geqslant \frac{\nu \inf _{\Omega^{\prime}}^{2} \psi_{0}}{\left|\Omega^{\prime}\right|\|\psi\|_{L_{2}(\Omega)}^{2}}\left\|\nabla \frac{\psi}{\psi_{0}}\right\|_{L_{1}\left(\Omega^{\prime}\right)}^{2}
$$

The last identity is the basic formula we use to estimate the spectral gaps. Before we give the proof of (5), we need to establish additional properties of the functions $\psi_{0}$ and $\psi$. This is the subject of the next two sections. The proof of (5) is given in Section 6. 


\section{Quantitative Harnack inequality}

In this section we prove the estimate

$$
\sup _{\widehat{\Omega}_{\frac{d}{8}}} \psi_{0} \leqslant C_{2} \inf _{\widehat{\Omega}_{\frac{d}{8}}} \psi_{0}
$$

with certain $C_{2}>0$. The constant $C_{2}$ depends only on the differential operator under consideration, and not on the particular non-negative (sub-) solution; see for instance the monographs [5, 12] for a proof of this statement.

In our context we need to know the explicit dependence of the constant $C_{2}$ on the parameters entering the definition of the differential operator. In the variants of Harnack's inequality (15) given in $[5,12]$ an explicit bound for $C_{2}$ is not obtained. We provide below a proof of Harnack's inequality along the lines of previos arguments, but which allows explicit control of the constants as functions of the various model paramters.

Theorem 6.1. Assume that $\varphi \in \mathcal{D}(\mathfrak{h})$ satisfies the inequalities

$$
(\varphi, \phi)_{L_{2}(\Omega)} \geq 0, \quad \mathfrak{h}[\varphi, \phi] \leq \lambda(\varphi, \phi)_{L_{2}(\Omega)}
$$

for all $\phi \in \mathcal{D}(\mathfrak{h})$ which are non-negative almost everywhere. Then there exists a constant $C_{2}$ such that

$$
\sup _{\widehat{\Omega}_{\frac{d}{8}}} \varphi \leqslant C_{2} \inf _{\widehat{\Omega}_{\frac{d}{8}}} \varphi
$$

The constant $C_{2}$ is given explicitly in (39).

Corollary 6.2. Let $C>0$ and $q>n$ be fixed. Let $V: \mathbb{R}^{n} \rightarrow \mathbb{R}$ be any potential such that for all $a \in \mathbb{R}^{n}$

$$
\|V\|_{L_{\frac{q}{2}}\left(B_{1}(a)\right)} \leq C .
$$

For $L \in \mathbb{N}$ and $x \in \mathbb{R}^{n}$ denote by $H_{\Lambda}=-\Delta+V$ the Schrödinger operator on $\Lambda:=\Lambda_{L}(x)=\left[-\frac{L}{2}, \frac{L}{2}\right]^{n}+x$ with periodic boundary conditions, by $\mathfrak{h}_{\Lambda}$ the associated quadratic form, and by $\lambda_{\Lambda}:=\inf \sigma\left(H_{\Lambda}\right)$. Then there exists a constant $0<C_{H}<\infty$, which depends on $q$ and $C$, but not on the particular choice of $V$, nor $L \in \mathbb{N}$, nor $x \in \mathbb{R}^{n}$ such that for any $\varphi \in \mathcal{D}\left(\mathfrak{h}_{\Lambda}\right)$ satisfying

$$
\begin{aligned}
& (\varphi, \phi)_{L_{2}(\Lambda)} \geq 0, \quad \text { and } \mathfrak{h}[\varphi, \phi] \leq \lambda_{\Lambda}(\varphi, \phi)_{L_{2}(\Lambda)} \\
& \text { for all } \phi \in \mathcal{D}\left(\mathfrak{h}_{\Lambda}\right) \text { which are non-negative a.e., }
\end{aligned}
$$

we have

$$
\sup _{\Lambda_{1}(y)} \varphi \leqslant C_{H} \inf _{\Lambda_{1}(y)} \varphi \quad \text { for any unit box } \quad \Lambda_{1}(y) \subset \Lambda_{L}(x)
$$


The remainder of this section is devoted to the proof of the theorem. The proof of the Harnack inequality in [5] is based on the Moser-iteration method. To make this iteraton work one needs first to provide a gradient estimate. This can be derived from the subsolution property. We present these arguments which lead up to the inequalities (17) and (18).

Let $\chi \in C_{0}^{\infty}(\Omega)$ be a cut-off function taking values in $[0,1]$. We introduce an auxilliary quadratic form

$$
\widetilde{\mathfrak{h}}[u, v]:=\sum_{i, j=1}^{n}\left(A_{i j} u_{i}, v_{j}\right)_{L_{2}(\Omega)}, \quad \widetilde{\mathfrak{h}}[u]:=\widetilde{\mathfrak{h}}[u, u]
$$

where $u=\left(u_{1}, \ldots, u_{n}\right), v=\left(v_{1}, \ldots, v_{n}\right), u_{i}, v_{i} \in \mathcal{D}(\mathfrak{h})$. In the second inequality of (16) we choose $\phi=\varphi^{\beta} \chi^{2}$ with $\beta \in \mathbb{R} \backslash\{0\}$ and obtain

$$
\widetilde{\mathfrak{h}}\left[\varphi^{\frac{\beta-1}{2}} \chi \nabla \varphi\right]=-\frac{2}{\beta} \widetilde{\mathfrak{h}}\left[\varphi^{\frac{\beta-1}{2}} \chi \nabla \varphi, \varphi^{\frac{\beta+1}{2}} \nabla \chi\right]+\frac{1}{\beta}\left(W_{0} \chi \varphi^{\frac{\beta+1}{2}}, \chi \varphi^{\frac{\beta+1}{2}}\right)_{L_{2}(\Omega)},
$$

where $W_{0}:=\lambda_{0}-V$. By the Cauchy-Schwarz inequality and the symmetry of the matrix $\left(A_{i j}\right)$ we have

$$
\begin{aligned}
\left|2 \widetilde{\mathfrak{h}}\left[\varphi^{\frac{\beta-1}{2}} \chi \nabla \varphi, \varphi^{\frac{\beta+1}{2}} \nabla \chi\right]\right| & \leqslant 2\left(\widetilde{\mathfrak{h}}\left[\varphi^{\frac{\beta-1}{2}} \chi \nabla \varphi\right]\right)^{\frac{1}{2}}\left(\widetilde{\mathfrak{h}}\left[\varphi^{\frac{\beta+1}{2}} \nabla \chi\right]\right)^{\frac{1}{2}} \\
& \leqslant \frac{|\beta|}{2} \widetilde{\mathfrak{h}}\left[\varphi^{\frac{\beta-1}{2}} \chi \nabla \varphi\right]+\frac{2}{|\beta|} \widetilde{\mathfrak{h}}\left[\varphi^{\frac{\beta+1}{2}} \nabla \chi\right] .
\end{aligned}
$$

The combination of the two last estimates yields

$$
\widetilde{\mathfrak{h}}\left[\varphi^{\frac{\beta-1}{2}} \chi \nabla \varphi\right] \leqslant \frac{4}{|\beta|^{2}} \widetilde{\mathfrak{h}}\left[\varphi^{\frac{\beta+1}{2}} \nabla \chi\right]+\frac{2}{|\beta|}\left(\left|W_{0}\right| \chi \varphi^{\frac{\beta+1}{2}}, \chi \varphi^{\frac{\beta+1}{2}}\right)_{L_{2}(\Omega)} .
$$

It is convenient to introduce the following auxiliary function,

$$
u:= \begin{cases}\varphi^{\frac{\beta+1}{2}}, & \beta \neq-1 \\ \ln \varphi, & \beta=-1\end{cases}
$$

Then the last inequality and (1) imply

$$
\begin{aligned}
\|\chi \nabla u\|_{L_{2}(\Omega)}^{2} & \leqslant C_{3}(\beta)\left(\|u \nabla \chi\|_{L_{2}(\Omega)}^{2}+\frac{|\beta|}{2 \mu}\left(\left|W_{0}\right| \chi u, \chi u\right)_{L_{2}(\Omega)}\right), \text { if } \beta \neq-1, \\
C_{3}(\beta) & :=\frac{(\beta+1)^{2}}{\beta^{2}} \frac{\mu}{\nu} \\
\|\chi \nabla u\|_{L_{2}(\Omega)}^{2} & \leqslant \frac{4 \mu}{\nu}\|\nabla \chi\|_{L_{2}(\Omega)}^{2}+\frac{2}{\nu}\left(\left|W_{0}\right| \chi, \chi\right)_{L_{2}(\Omega)}, \quad \text { if } \beta=-1 .
\end{aligned}
$$


An interpolation inequality for Sobolev spaces (see e.g. [12, Chapter II, (2.9)]) implies $\|\chi u\|_{L_{2 p}(\Omega)}^{2} \leqslant(p+1)^{2}\|\nabla(\chi u)\|_{L_{2}(\Omega)}^{2}$. Now estimate (17) yields

$$
\begin{aligned}
\|\chi u\|_{L_{2 p}(\Omega)}^{2} & \leqslant 2(p+1)^{2}\left(\|\chi \nabla u\|_{L_{2}(\Omega)}^{2}+\|u \nabla \chi\|_{L_{2}(\Omega)}^{2}\right) \\
& \leqslant 2(p+1)^{2}\left(1+C_{3}(\beta)\right)\|u \nabla \chi\|_{L_{2}(\Omega)}^{2}+\frac{(p+1)^{2} C_{3}(\beta)|\beta|}{\mu}\left(\left|W_{0}\right| \chi u, \chi u\right)_{L_{2}(\Omega)}
\end{aligned}
$$

where, we remind, the number $p$ was introduced in (4). Denote $\hat{q}:=\frac{q}{q-2}$. We employ the Hölder inequality and arrive at the estimate

$$
\begin{aligned}
\|\chi u\|_{L_{2 p}(\Omega)}^{2} \leqslant & 2(p+1)^{2}\left(1+C_{3}(\beta)\right)|\operatorname{supp} \chi|^{\frac{2}{q}}\|u \nabla \chi\|_{L_{2 \hat{q}}(\Omega)}^{2} \\
& +\frac{(p+1)^{2} C_{3}(\beta)|\beta|}{\mu}\left\|W_{0}\right\|_{L_{\frac{q}{2}}(\operatorname{supp} \chi)}\|\chi u\|_{L_{2 \hat{q}}(\Omega)}^{2} .
\end{aligned}
$$

Now we choose the function $\chi$ more specifically. Let $0<\gamma_{1}<\gamma_{2}<\frac{1}{4}, a \in \widehat{\Omega}$, and let

$$
\chi \equiv 1 \quad \text { in } B_{\gamma_{1} d}(a), \quad \chi \equiv 0 \quad \text { outside } B_{\gamma_{2} d}(a), \quad\|\nabla \chi\|_{\infty} \leqslant \frac{2}{\left(\gamma_{2}-\gamma_{1}\right) d}
$$

Then

$$
\begin{aligned}
&\|u\|_{L_{2 p}\left(B_{\gamma_{1}}(a)\right)}^{2} \leqslant C_{4}\left(\gamma_{2}-\gamma_{1}, \beta\right)\|u\|_{L_{2 \hat{q}}\left(B_{\gamma_{2} d}(a)\right)}^{2}, \\
& C_{4}\left(\gamma_{2}-\gamma_{1}, \beta\right):=\frac{8(p+1)^{2}\left(1+C_{3}(\beta)\right) \Theta_{n}^{\frac{2}{q}} d^{\frac{2 n}{q}-2}}{4^{\frac{2 n}{q}}\left(\gamma_{2}-\gamma_{1}\right)^{2}}+\frac{(p+1)^{2} C_{3}(\beta)|\beta|}{\mu}\left\|W_{0}\right\|_{L_{\frac{q}{2}}\left(B_{\frac{d}{4}}(a)\right)} .
\end{aligned}
$$

Thanks to the abbreviation

$$
\Phi[b, \gamma]:=\left(\int_{B_{\gamma d}(a)}|\varphi|^{b} \mathrm{~d} x\right)^{\frac{1}{b}}, \quad b \in \mathbb{R} \backslash\{0\}, \quad \gamma \in\left(0, \frac{1}{2}\right),
$$

inequality (19) can be rewritten as

$$
\Phi\left[p(\beta+1), \gamma_{1}\right] \leqslant C_{4}^{\frac{1}{\beta+1}}\left(\gamma_{2}-\gamma_{1}, \beta\right) \Phi\left[\hat{q}(\beta+1), \gamma_{2}\right], \quad \text { if } \beta+1>0
$$

and

$$
\Phi\left[\hat{q}(\beta+1), \gamma_{2}\right] \leqslant C_{4}^{-\frac{1}{\beta+1}}\left(\gamma_{2}-\gamma_{1}, \beta\right) \Phi\left[p(\beta+1), \gamma_{1}\right], \quad \text { if } \beta+1<0 .
$$

Now we can start the iteration procedure mentioned above. For this purpose we fix a positive number $t$ and choose a sequence of length scales $\tau_{m}$ and exponents $\beta_{n}, m \in \mathbb{N}$, as follows

$$
\tau_{m}:=\frac{1}{8}+\frac{1}{2^{m+2}}, \quad \beta:=\beta_{m}:=\left(\frac{p}{\hat{q}}\right)^{m} \frac{t}{p}-1, \quad m \in \mathbb{N} .
$$


Recall that by definition $p>\hat{q}$ and choose $t \geqslant 2 p$. This ensures that $\beta_{m} \geqslant 1$ for all $m \in \mathbb{N}$. The last inequality, Lemma 4.1 and the definitions of $p, C_{3}, C_{4}$, and $\widehat{V}$ imply

$$
\frac{p}{\hat{q}} \leqslant 3, \quad|\beta| \leqslant \frac{3^{m} t}{p}, \quad C_{3}(\beta) \leqslant \frac{4 \mu}{\nu}, \quad C_{4}\left(\tau_{m+1}-\tau_{m+2}, \beta_{m}\right) \leqslant \frac{4^{m} t}{p} C_{5},
$$

where

$$
C_{5}:=(p+1)^{2}\left(2^{11}\left(1+\frac{4 \mu}{\nu}\right) \frac{\Theta_{n}^{\frac{2}{q}} d^{\frac{2 n}{q}-2}}{4^{\frac{2 n}{q}}}+4 \frac{\widehat{V}}{\nu}\right) .
$$

Hence, inequality (20) yields

$$
\begin{aligned}
\Phi\left[t\left(\frac{p}{\hat{q}}\right)^{m}, \tau_{m+2}\right] & \leqslant\left(\frac{4^{m} C_{5} t}{p}\right)^{\frac{p}{t}\left(\frac{\hat{q}}{p}\right)^{m}} \Phi\left[t\left(\frac{p}{\hat{q}}\right)^{m-1}, \tau_{m+1}\right] \\
& \leqslant \prod_{i=1}^{m}\left(\frac{4^{i} C_{5} t}{p}\right)^{\frac{p}{t}\left(\frac{\hat{q}}{p}\right)^{i}} \Phi\left[t, \tau_{2}\right],
\end{aligned}
$$

for all $m \geqslant 1$. Direct calculations show $\sum_{i=1}^{\infty}\left(\frac{\hat{q}}{p}\right)^{i}=\frac{\hat{q}}{p-\hat{q}}, \sum_{i=1}^{\infty} i\left(\frac{\hat{q}}{p}\right)^{i}=\frac{p \hat{q}}{(p-\hat{q})^{2}}$, $\max _{[2 p,+\infty)} \frac{\ln t}{t} \leqslant \max _{[2,+\infty)} \frac{\ln t}{t}=\mathrm{e}^{-1}, t^{\frac{1}{t}} \leqslant \mathrm{e}^{-\frac{1}{\mathrm{e}}}<2$. We pass in (24) to the limit $m \rightarrow+\infty$. Then [5, Problem 7.1] and the monotonicity of $\Phi[b, \gamma]$ with respect to the radius $\gamma$ imply

$$
\begin{aligned}
\sup _{B_{\frac{d}{8}}(a)} \varphi & =\lim _{m \rightarrow+\infty} \Phi\left[t\left(\frac{p}{\hat{q}}\right)^{m}, \tau_{m+2}\right] \leqslant C_{6} \Phi\left[t, \frac{3}{16}\right], \\
C_{6} & :=2^{\frac{p \hat{q}}{p-\hat{q}}+\frac{p \hat{q}}{(p-\hat{q})^{2}}}\left(\frac{C_{5}}{p}\right)^{\frac{p \hat{q}}{t(p-\hat{q})}} .
\end{aligned}
$$

Thus we are able to bound the supremum of $\varphi$ in a small ball by some $L_{p}$-norm with a finite exponent on a larger ball. Similarly, it is possible to give a lower bound on the infimum of $\varphi$ by some $L_{p}$-norm. For this purpose we consider now the parameter range $\beta+1<0$. In this case two last estimates in (23) remain true. We chose a different sequence of scales than in (22). More precisely, fix an arbitrary positive $t$ and set

$$
\beta:=\beta_{m}:=-\frac{t}{\hat{q}}\left(\frac{p}{\hat{q}}\right)^{m}-1, \quad m \in \mathbb{Z}_{+} .
$$

We observe that by (19) and the first estimate in (23)

$$
C_{4}\left(\tau_{m+1}-\tau_{m+2}, \beta_{m}\right) \leqslant 4^{m}\left(1+\frac{t}{\hat{q}}\right) C_{7}, \quad C_{7}:=(p+1)^{2}\left(2^{11}\left(1+\frac{\mu}{\nu}\right) \frac{\Theta_{n}^{\frac{2}{q}} d^{\frac{2 n}{q}-2}}{4^{\frac{2 n}{q}}}+\frac{\widehat{V}}{\nu}\right) .
$$


Then estimate (21) implies

$$
\begin{aligned}
\Phi\left[-t, \tau_{1}\right] & \leqslant\left(4^{0} C_{7} \cdot\left(\frac{t}{\hat{q}}+1\right)\right)^{\frac{\hat{q}}{t}} \Phi\left[-\frac{p t}{\hat{q}}, \tau_{2}\right] \\
& \leqslant\left(4^{0} C_{7} \cdot\left(\frac{t}{\hat{q}}+1\right)\right)^{\frac{\hat{q}}{t}}\left(4^{1} C_{7} \cdot\left(\frac{t}{\hat{q}}+1\right)\right)^{\frac{\hat{q}^{2}}{p t}} \Phi\left[-\frac{p^{2} t}{\hat{q}^{2}}, \tau_{3}\right] \\
& \leqslant \prod_{i=1}^{m}\left(4^{i-1} C_{7}\left(\frac{t}{\hat{q}}+1\right)\right)^{\frac{\hat{q}}{t}\left(\frac{\hat{q}}{p}\right)^{i-1}} \Phi\left[-\frac{p^{m} t}{\hat{q}^{m}}, \tau_{m}\right] .
\end{aligned}
$$

Again we pass to the limit $m \rightarrow+\infty$ and use [5, Problem 7.1] to arrive at the identity

$$
\Phi\left[-t, \frac{1}{4}\right] \leqslant C_{8}(t) \inf _{B_{\frac{d}{8}}(a)} \varphi, \quad C_{8}(t):=2^{\frac{\hat{q}^{2}}{(p-\hat{q})^{2}}}\left(C_{7}\left(\frac{t}{\hat{q}}+1\right)\right)^{\frac{p \hat{q}}{t(p-\hat{q})}} .
$$

Now we have to cover the intermediate parameter region $0<b \leq 2 p$ for the exponent in $\Phi[b, \gamma]$. For this purpose let us return back to inequality (18). We fix $a \in \widehat{\Omega}, \rho \leqslant \frac{d}{4}$, and choose $\chi$ such that $\chi \equiv 1$ in $B_{\rho}(a), \chi \equiv 0$ outside $B_{2 \rho}(a)$, and $\|\nabla \chi\|_{\infty} \leqslant 2 \rho^{-1}$. It follows from (18) and Hölder inequality that

$$
\begin{aligned}
& \|\nabla u\|_{L_{2}\left(B_{\rho}(a)\right)}^{2} \leqslant 2^{n+4} \mu \nu^{-1} \Theta_{n} \rho^{n-2}+2^{1+\frac{n}{\hat{q}}} \nu^{-1} \Theta_{n}^{\frac{1}{\hat{q}}} \rho^{\frac{n}{\hat{q}}}\left\|W_{0}\right\|_{L_{\frac{q}{2}}\left(B_{2 \rho}(a)\right)}, \\
& \|\nabla u\|_{L_{1}\left(B_{\rho}(a)\right)}^{2} \leqslant \Theta_{n} \rho^{n}\|\nabla u\|_{L_{2}\left(B_{\rho}(a)\right)}^{2} .
\end{aligned}
$$

Thus we have established that

$$
\|\nabla u\|_{L_{1}\left(B_{\rho}(a)\right)} \leqslant C_{9} \rho^{n-1}, \quad C_{9}:=\Theta_{n}^{\frac{1}{2}} \nu^{-\frac{1}{2}}\left(2^{n+4} \mu \Theta_{n}+2^{n\left(1+\frac{2}{q}\right)-3} \widehat{V} \Theta_{n}^{\frac{1}{\hat{q}}} d^{2\left(1-\frac{n}{q}\right)}\right)^{\frac{1}{2}} .
$$

If we take any $\widetilde{\rho} \leq \rho$ and a ball $B_{\widetilde{\rho}}(\widetilde{a})$ contained in $\Omega$, then $\|\nabla u\|_{L_{1}\left(B_{\rho}(a) \cap B_{\tilde{\rho}}(\widetilde{a})\right)} \leqslant$ $C_{9} \widetilde{\rho}^{n-1}$. This shows that the function $|\nabla u|$ is in the Morrey class, cf. [5, Section 7.9], and that the corresponding norm is bounded by $C_{9}$.

Using this estimate, [5, Chapter 7, Section 7.8, Lemma 7.16], the inequalities $1<\frac{n}{n-1} \leqslant 2, \sum_{j=1}^{\infty} \frac{j^{j}}{j !(2 \mathrm{e})^{j}}<\frac{1}{3}$, and analysing the proof of [5, Chapter 7 , Section 7.9, Lemma 7.20], one can make sure that

$$
\begin{gathered}
\int_{B_{\rho}(a)} \exp \left(C_{10}\left|u(x)-u_{\rho}\right|\right) \mathrm{d} x \leqslant C_{11} \rho^{n}, \\
u_{\rho}:=\frac{1}{\left|B_{\rho}(a)\right|} \int_{B_{\rho}(a)} u(x) \mathrm{d} x, \quad C_{10}=\frac{\Theta_{n}}{2^{n+1} \mathrm{e} C_{9}}, \quad C_{11}=2^{n+1} \Theta_{n} .
\end{gathered}
$$


Hence,

$$
\begin{array}{r}
\int_{B_{\rho}(a)} \exp \left( \pm C_{10} u(x)\right) \mathrm{d} x \leqslant C_{11} \rho^{n} \exp \left( \pm C_{10} u_{\rho}\right), \\
\int_{B_{\rho}(a)} \exp \left(C_{10} u(x)\right) \mathrm{d} x \int_{B_{\rho}(a)} \exp \left(-C_{10} u(x)\right) \mathrm{d} x \leqslant C_{11}^{2} \rho^{2 n}
\end{array}
$$

that yields

$$
\int_{B_{\rho}(a)} \exp \left(C_{10} u(x)\right) \mathrm{d} x \leqslant C_{11}^{2} \rho^{2 n}\left(\int_{B_{\rho}(a)} \exp \left(-C_{10} u(x)\right) \mathrm{d} x\right)^{-1}
$$

We replace $u$ by $\ln \varphi$ and obtain $\Phi\left[C_{10}, \frac{\rho}{d}\right] \leqslant\left(C_{11} \rho^{n}\right)^{\frac{2}{C_{10}}} \Phi\left[-C_{10}, \frac{\rho}{d}\right]$. The relevance of the last estimate is that it relates $\Phi[b, \gamma]$ with positive and negative values of $b$ each to other. The problem is however that we know only that $b=C_{10}$ is positive, but a close look reveals that it smaller than $2 p$, the parameter value for which inequality $(25)$ is valid. Indeed, $p>1$, and

$$
C_{9} \geqslant 2^{\frac{n}{2}+2} \Theta_{n}\left(\frac{\mu}{\nu}\right)^{\frac{1}{2}} \geqslant 2^{\frac{n}{2}+2} \Theta_{n}, \quad C_{10}<2^{-\frac{3 n}{2}-3}<1 .
$$

For this reason we have to bridge the gap between the parameter value $C_{10}$ and $2 p$.

We let $\rho=\frac{d}{4}$, and use (27) with $t=C_{10}$. This implies

$$
\Phi\left[C_{10}, \frac{1}{4}\right] \leqslant\left(\frac{C_{11} d^{n}}{4^{n}}\right)^{\frac{2}{C_{10}}} C_{8}\left(C_{10}\right) \inf _{B_{\frac{d}{8}}(a)} \varphi .
$$

We introduce a sequence

$$
z_{k}:=\frac{2 \hat{q}^{2}}{p+\hat{q}}\left(\frac{\hat{q}}{p}\right)^{k}, \quad k \geqslant 0 .
$$

Let $l$ be the minimal index in this sequence such that $z_{l} \leqslant C_{10}$, i.e., $l$ is the minimal nonnegative integer greater than or equal to $-\log _{\frac{p}{\tilde{q}}} \frac{C_{10}(p+\hat{q})}{2 \hat{q}^{2}}$. The relations (4) and (28) imply

$$
\begin{array}{ll}
\frac{C_{10}(p+\hat{q})}{2 \hat{q}^{2}}<\frac{2 \hat{q}+1}{2^{7} \hat{q}^{2}}<\frac{3}{2^{7}}, & n=2 \\
\frac{C_{10}(p+\hat{q})}{2 \hat{q}^{2}}<C_{10} p \quad<\frac{n}{2^{\frac{3 n}{2}+3}(n-2)}<\frac{3}{2^{\frac{3 n}{2}+3}} \leqslant \frac{3}{2^{7}}, & n>2 .
\end{array}
$$

Hence, $-\log _{\frac{p}{\hat{q}}} \frac{C_{10}(p+\hat{q})}{2 \hat{q}^{2}}>0$, and it follows from the definition of $l$ that

$$
l=-\log _{\frac{p}{\hat{q}}} \frac{C_{10}(p+\hat{q})}{2 \hat{q}^{2}}+\eta
$$


where $\eta \in[0,1)$. Thus,

$$
C_{10} \frac{\hat{q}}{p}<z_{l} \leqslant C_{10}
$$

By Hölder inequality we obtain

$$
\Phi\left[z_{l}, \frac{1}{4}\right] \leqslant\left(\frac{\Theta_{n} d^{n}}{4^{n}}\right)^{\frac{C_{10}-z_{l}}{C_{10} z_{l}}} \Phi\left[C_{10}, \frac{1}{4}\right] .
$$

Our next aim is to estimate $\Phi\left[t, \frac{3}{16}\right]$ by $\Phi\left[z_{l}, \frac{1}{4}\right]$ for some $t \geqslant 2 p$. This will be again done by an interation, but this time it will have only a finite number of steps. We introduce the number $N$ as the minimal integer greater than or equal to

$$
\log _{\frac{p}{\hat{q}}} \frac{2 p}{z_{l}}=l+1+\log _{\frac{p}{\hat{q}}} \frac{p+\hat{q}}{\hat{q}} .
$$

The identity (31) implies the upper bound for $N$,

$$
N \leqslant l+2+\log _{\frac{p}{\hat{q}}} \frac{p+\hat{q}}{\hat{q}} \leqslant \log _{\frac{p}{\hat{q}}} \frac{p+\hat{q}}{\hat{q}}-\log _{\frac{p}{\hat{q}}} \frac{C_{10}(p+\hat{q})}{2 \hat{q}^{2}}+3 \leqslant \log _{\frac{p}{\hat{q}}} \frac{2 p^{3}}{C_{10} \hat{q}^{2}} .
$$

It follows from the definition of $z_{l}$ that

$$
\max _{m \in \mathbb{N}}\left|\frac{z_{l}}{\hat{q}}\left(\frac{p}{\hat{q}}\right)^{m}-1\right|^{-1}=\left|\frac{z_{l}}{\hat{q}}\left(\frac{p}{\hat{q}}\right)^{l}-1\right|^{-1}=\frac{p+\hat{q}}{p-\hat{q}}
$$

This time it turns out to be convenient to choose the sequences of length scales $\widetilde{\tau}_{m}$ and exponents $\beta_{m}, m \in \mathbb{N}$, according to

$$
\widetilde{\tau}_{m}=\frac{1}{4}-\frac{m}{16 N}, \quad \beta:=\beta_{m}:=\frac{z_{l}}{\hat{q}}\left(\frac{p}{\hat{q}}\right)^{m}-1 .
$$

By (28), (32), (35), (36), (34) and the definition of $C_{3}$ and $C_{4}$ we obtain

$$
\begin{gathered}
C_{3}(\beta) \leqslant \frac{(|\beta|+1)^{2}}{|\beta|^{2}} \frac{\mu}{\nu}=\left(1+\frac{1}{|\beta|}\right)^{2} \frac{\mu}{\nu} \leqslant \frac{4 p^{2}}{(p-\hat{q})^{2}} \frac{\mu}{\nu}, \\
C_{4}\left(\widetilde{\tau}_{m}-\widetilde{\tau}_{m-1}, \beta_{m}\right) \leqslant C_{12}\left(\frac{p}{\hat{q}}\right)^{m}, \\
C_{12}:=\frac{4(p+1)^{2} p^{2}}{(p-\hat{q})^{2}} \frac{\widehat{V}}{\nu}\left(1+\frac{C_{10}}{\hat{q}}\right) \\
+2^{11-\frac{4 n}{q}}(p+1)^{2} \Theta_{n}^{\frac{2}{q}} d^{\frac{2 n}{q}-2}\left(1+\frac{4 p^{2}}{(p-\hat{q})^{2}} \frac{\mu}{\nu}\right) \log _{\frac{p}{\hat{q}}}^{2} \frac{2 p^{3}}{C_{10} \hat{q}^{2}} .
\end{gathered}
$$


Taking these relations and (32) into account, we apply the estimates (20),

$$
\begin{aligned}
& \Phi\left[z_{l}\left(\frac{p}{\hat{q}}\right)^{N}, \frac{3}{16}\right]=\Phi\left[z_{l}\left(\frac{p}{\hat{q}}\right)^{N}, \widetilde{\tau}_{N}\right] \\
& \leqslant\left(C_{12}\left(\frac{p}{\hat{q}}\right)^{N-1}\right)^{\frac{\hat{q}}{z_{l}}\left(\frac{\hat{q}}{p}\right)^{N-1}} \Phi\left[z_{l}\left(\frac{p}{\hat{q}}\right)^{N-1}, \widetilde{\tau}_{N-1}\right] \\
& \leqslant\left(C_{12} \frac{\hat{q}}{p}\left(\frac{p}{\hat{q}}\right)^{N}\right)^{\frac{p}{z_{l}}\left(\frac{\hat{q}}{p}\right)^{N}}\left(C_{12} \frac{\hat{q}}{p}\left(\frac{p}{\hat{q}}\right)^{N-1}\right)^{\frac{p}{z_{l}}\left(\frac{\hat{q}}{p}\right)^{N-1}} \Phi\left[z_{l}\left(\frac{p}{\hat{q}}\right)^{N-2}, \widetilde{\tau}_{N-2}\right] \\
& \leqslant \prod_{i=1}^{N}\left(C_{12} \frac{\hat{q}}{p}\left(\frac{p}{\hat{q}}\right)^{i}\right)^{\frac{p}{z_{l}}\left(\frac{\hat{q}}{p}\right)^{i}} \Phi\left[z_{l}, \widetilde{\tau}_{0}\right] \leqslant\left(\frac{p}{\hat{q}}\right)^{\frac{p^{2} \hat{q}}{z_{l}(p-\hat{q})^{2}}}\left(C_{12} \frac{\hat{q}}{p}\right)^{\frac{p \hat{q}}{z_{l}(p-\hat{q})}} \Phi\left[z_{l}, \frac{1}{4}\right] \\
& \leqslant\left(\frac{p}{\hat{q}}\right)^{\frac{p^{2} \hat{q}}{C_{10}(p-\hat{q})^{2}}}\left(C_{12} \frac{\hat{q}}{p}\right)^{\frac{p \hat{q}}{z_{l}(p-\hat{q})}} \Phi\left[z_{l}, \frac{1}{4}\right] .
\end{aligned}
$$

Note that $z_{l}\left(\frac{p}{\hat{q}}\right)^{N} \geq 2 p$ by (34). We choose $t=z_{l}\left(\frac{p}{\hat{q}}\right)^{N}$ and combine the obtained inequality with (25), (29), and (33),

$$
\begin{gathered}
\sup _{B_{\frac{d}{8}}(a)} \varphi \leqslant C_{13} \inf _{B_{\frac{d}{8}}(a)} \varphi \\
C_{13}:=2^{\frac{p \hat{q}}{p-\hat{q}}+\frac{p \hat{q}+\hat{q}^{2}}{(p-\hat{q})^{2}}-\frac{2(n-1)}{C_{10}}\left(\frac{p}{\hat{q}}\right)^{\frac{p^{2} \hat{q}}{C_{10}(p-\hat{q})^{2}}}\left(1+\frac{2 p^{2}}{\hat{q}^{2}}\right)^{\frac{\hat{q}}{2(p-\hat{q})}} \max \left\{\left(\Theta_{n} d^{n}\right)^{\frac{2}{C_{10}}},\left(\Theta_{n} d^{n}\right)^{\frac{p+\hat{q}}{C_{10} \hat{q}}}\right\}} \\
\cdot \max \left\{\left(\frac{C_{5} C_{7}}{p}\right)^{\frac{\hat{q}}{2(p-\hat{q})}},\left(\frac{C_{5} C_{7}}{p}\right)^{\frac{\hat{q}^{2}}{2 p(p-\hat{q})}}\right\} \max \left\{\left(\frac{C_{12} \hat{q}}{p}\right)^{\frac{p \hat{q}}{C_{10}(p-\hat{q})}},\left(\frac{C_{12} \hat{q}}{p}\right)^{\frac{p^{2}}{C_{10}(p-\hat{q})}}\right\}
\end{gathered}
$$

for all $a \in \widehat{\Omega}$, where we have used that by (28), (32), (35), (30), and $t \geqslant 2 p$ $2 p \leqslant t=z_{l}\left(\frac{p}{\hat{q}}\right)^{N} \leqslant \frac{2 \hat{q}^{2}}{p+\hat{q}}\left(\frac{p}{\hat{q}}\right)^{N-l} \leqslant \frac{2 p^{2}}{\hat{q}}$,

$$
\begin{aligned}
& \left(\frac{\Theta_{n} d^{n}}{4^{n}}\right)^{\frac{C_{10}-z_{l}}{C_{10} z_{l}}}\left(\frac{C_{11} \Theta_{n} d^{n}}{4^{n}}\right)^{\frac{2}{C_{10}}}=\frac{\left(\Theta_{n} d^{n}\right)^{\frac{1}{C_{10}}+\frac{1}{z_{l}}}}{2^{\frac{2 n}{z_{l}}-\frac{2}{C_{10}}}} \\
& \leqslant 2^{-\frac{2(n-1)}{C_{10}}} \max \left\{\left(\Theta_{n} d^{n}\right)^{\frac{2}{C_{10}}},\left(\Theta_{n} d^{n}\right)^{\frac{p+\hat{q}}{C_{10}}}\right\}, \\
& \left(\frac{C_{5} C_{7}}{p}\right)^{\frac{p \hat{q}}{t(p-\hat{q})}} \leqslant \max \left\{\left(\frac{C_{5} C_{7}}{p}\right)^{\frac{\hat{q}}{2(p-\hat{q})}},\left(\frac{C_{5} C_{7}}{p}\right)^{\frac{\hat{q}^{2}}{2 p(p-\hat{q})}}\right\}, \\
& \left(\frac{C_{12} \hat{q}}{p}\right)^{\frac{p \hat{q}}{z_{l}(p-\hat{q})}} \leqslant \max \left\{\left(\frac{C_{12} \hat{q}}{p}\right)^{\frac{p \hat{q}}{C_{10}(p-\hat{q})}},\left(\frac{C_{12} \hat{q}}{p}\right)^{\frac{p^{2}}{C_{10}(p-\hat{q})}}\right\} .
\end{aligned}
$$


We observe that

$$
C_{13} \geqslant 1
$$

otherwise inequality (37) is impossible.

Let $x_{\max } \in \widehat{\Omega}_{\frac{d}{8}}$ be the point of global maximum of $\varphi$ in $\widehat{\Omega}_{\frac{d}{8}}$, and $x_{\text {min }}$ be the point of global minimum in the same domain. Then there exists two points $a_{ \pm} \in \widehat{\Omega}$ such that $x_{\max } \in \bar{B}_{\frac{d}{8}}\left(a_{+}\right), x_{\min } \in \bar{B}_{\frac{d}{8}}\left(a_{-}\right)$. We connect the points $a_{+}$ and $a_{-}$by an admissible cylinder of the length at most $L$; the corresponding curve lies in $\widehat{\Omega}$. We cover the corresponding curve by balls of radius $\frac{d}{8}$; the balls should have at least common boundary points, and two of these balls must be $B_{\frac{d}{8}}\left(a_{ \pm}\right)$. It is clear that it is possible to cover the mentioned curve by at most $\frac{4 L}{d}$ balls. We apply estimate (37) to each of these balls and proceed as in the proof of [5, Chapter II, Section 2.3, Theorem 2.5], that leads us to the estimate (15), where

$$
C_{2}=C_{13}^{\frac{4 L}{d}}
$$

\section{Estimates for the second eigenfunction $\psi$}

In this section we study properties of an eigenfunction $\psi$ associated to an eigenvalue $\lambda \in\left(\lambda_{0}, 0\right)$. In particular, we establish a relation between the supremum-, the Hölder- and $L_{2}$-norms of $\psi$.

Lemma 7.1. There exist a point $x_{-} \in \Omega_{0}$ such that $\psi\left(x_{-}\right)=0$.

Proof. We prove the existence by contradiction. Suppose that such a point $x_{-}$ does not exist and consider the domain $\Omega_{-}:=\{x \in \Omega: \psi(x) \leqslant 0\}$. Lemma 5.1 yields that this domain is closed and by the assumption $\Omega_{-} \cap \Omega_{0}=\varnothing$. Now we restrict the quadratic form $\mathfrak{h}$ to the subspace $\stackrel{\circ}{W}_{2}^{1}\left(\Omega_{-}, \Gamma \cap \partial \Omega_{-}\right) \cap L_{2}\left(\Omega_{-} ; V^{+}\right)$. This form is closed, symmetric and lower-semibounded. By $\mathcal{H}_{-}$we denote the associated self-adjoint operator in $L_{2}\left(\Omega_{-}\right)$. It follows from the identity $\Omega_{-} \cap \Omega_{0}=\varnothing$ and the definition of the function $\psi$ that it belongs to $\stackrel{\circ}{W}_{2}^{1}\left(\Omega_{-}, \Gamma \cap \partial \Omega_{-}\right) \cap L_{2}\left(\Omega_{-} ; V^{+}\right)$and is a generalized solution to the equation

$$
\left(-\sum_{i, j=1}^{n} \frac{\partial}{\partial x_{i}} A_{i j} \frac{\partial}{\partial x_{j}}+V-\lambda\right) \psi=0 \quad \text { in } \Omega_{-}
$$

satisfying Dirichlet boundary condition on $\Gamma \cap \partial \Omega_{-}$, and the Neumann condition on the rest of the boundary. Therefore, it is an eigenfunction of this operator associated with an eigenvalue $\lambda<0$. By Dirichlet-Neumann bracketing and (3c) it follows that $\inf \sigma\left(\mathcal{H}_{-}\right) \geqslant \inf \sigma\left(\mathcal{H}_{0}\right) \geqslant 0$, which yields a contradiction. Therefore, $\Omega_{-} \cap \Omega_{0} \neq \varnothing$, i.e., there exists $x_{-} \in \Omega_{0}$ such that $\psi\left(x_{-}\right)=0$. 
Having Lemma 5.1 in mind and, if needed, changing the sign of $\psi$, we normalize the function $\psi$ by the requirement

$$
\max _{\bar{\Omega}_{0}}|\psi|=\max _{\bar{\Omega}_{0}} \psi=1
$$

The function $\psi$ is a generalized solution to the equation

$$
\left(-\sum_{i, j=1}^{n} \frac{\partial}{\partial x_{i}} A_{i j} \frac{\partial}{\partial x_{j}}+V^{+}-\lambda\right) \psi=0 \quad \text { in } \Omega \backslash \Omega_{0} .
$$

Due to (3c) we have $V^{+}-\lambda \geqslant 0$ in $\Omega \backslash \Omega_{0}$. Together with the fact that $\psi$ vanishes on $\partial \Omega$, by the weak maximum principle (see [5, Chapter 8 , Section 8.1 , Theorem 8.1]) and (40) we have the estimate $|\psi(x)| \leqslant \max _{\partial \Omega_{0}}|\psi| \leqslant 1, x \in \overline{\Omega \backslash \Omega_{0}}$. By (40) it yields

$$
\max _{\bar{\Omega}}|\psi|=1
$$

Lemma 7.2. For each ball $B_{r}(a), a \in \Omega_{0}, r \leqslant r_{1}$, the inequality

$$
|\psi(x)-\psi(y)| \leqslant \frac{C_{14} r^{\alpha}}{r_{1}^{\alpha}}, \quad x, y \in \bar{B}_{r}(a)
$$

holds true, where

$$
\begin{aligned}
r_{1} & :=\left\{\Theta_{n}^{-\frac{1}{n}}\left(\frac{\nu}{12(p+1)^{2} \widehat{V}}\right)^{\frac{q}{2(q-n)}}, \frac{d}{4}\right\}, \\
\alpha & =\min \left\{-\log _{4}\left(1-2^{-C_{15}}\right), 1-\frac{n}{q}\right\}, \\
C_{14} & =4^{\alpha} \max \left\{2, \frac{2^{C_{15}+2} \nu}{9 \sqrt{6} \mu(p+1) \Theta_{n}^{\frac{1}{n}}}\right\}, \\
C_{15} & :=3+81 \cdot 2^{n+9}\left(\theta_{n}+1\right)^{2} n^{-2} \mu^{2} \nu^{-2} C_{16}^{\frac{2(n-1)}{n}}, \\
C_{16} & :=\max \left\{2^{2 n+1} \Theta_{n}^{-1}, 4^{\frac{q^{2} n^{2}}{(q-n)^{2}}} C_{17}^{\frac{q n}{q-n}}\right\}, \\
C_{17} & :=9 \cdot 2^{2 n+9} \Theta_{n^{\frac{1-q}{q}}} n^{-1}\left(\theta_{n}+1\right) \mu \nu^{-1} .
\end{aligned}
$$

Proof. The statement of this lemma was proven in [12, Chapter III, Section 13, Theorem 13.1], but the explicit formulae for $\alpha, r_{1}$ and $C_{14}$ were not given. For this reason we partially reproduce the proof to obtain the explicit formulae for the mentioned constants. The idea of the proof is to estimate the norm of the gradient of $\psi$ on some special sets. The resulting estimates guarantee that $\psi$ belongs to a certain class of functions which can be embedded into a 
Hölder space. The Hölder norm and exponent can be expressed explicitly via the constants in the estimates for the norm of the gradient.

We choose $\widetilde{a} \in \Omega_{0}$. We are going to prove that for an appropriate choice of the constants $C$ and $\widetilde{C}$ the function $\psi$ belongs to the special class of functions $\mathfrak{B}_{2}\left(B_{\frac{d}{4}}(\widetilde{a}), 1, C, \widetilde{C}, 1, \frac{1}{q}\right)$ defined in $[12$, Chapter II, Section 6]. It was shown in [12, Chapter II, Theorem 6.1] that this class is embedded in a Hölder space. Moreover, an explicit formula for the Hölder exponent and the estimate for the norm were given. This is why we need to estimate the constants $C, \widetilde{C}$ explicitly to apply the cited theorem.

Given any number $k \geqslant-2$ and any ball $B_{r}(a) \subset B_{\frac{d}{4}}(\widetilde{a})$, we consider the set $M_{k, r}:=\{x: \psi(x)>k\} \cap B_{r}(a)$. We only need to consider $k \geqslant-2$, since this is a superset of the values of $k$ which satisfy inequality (6.2) in [12, Chapter II], namely

$$
k \geqslant \max _{x \in B_{r}(a)} \pm \psi(x)-1
$$

We note that this inequality forms part of the definition of the functional class $\mathfrak{B}_{2}\left(B_{\frac{d}{4}}(\widetilde{a}), 1, C, \widetilde{C}, 1, \frac{1}{q}\right)$.

Let $\chi \in C^{\infty}\left(\overline{B_{r}(a)}\right)$ be a cut-off function taking values in $(0,1)$ and vanishing outside $B_{r}(a)$. The function $v(x):=\chi(x)^{2} \max \{\psi(x)-k ; 0\}$ belongs to $\mathcal{D}(\mathfrak{h})$ and since $\psi$ is a weak solution we have $\mathfrak{h}[\psi, v]-\lambda(\psi, v)_{L_{2}(\Omega)}=0$. We substitute this relation and the definition of vinto the formula for $\mathfrak{h}$,

$$
\begin{aligned}
\sum_{i, j=1}^{n} \int_{M_{k, r}} A_{i j} \frac{\partial \psi}{\partial x_{i}} \frac{\partial \psi}{\partial x_{j}} \chi^{2} \mathrm{~d} x= & -2 \sum_{i, j=1}^{n} \int_{M_{k, r}} A_{i j} \frac{\partial \psi}{\partial x_{i}} \frac{\partial \chi}{\partial x_{j}}(\psi-k) \chi \mathrm{d} x \\
& +\int_{M_{k, r}} W(\psi-k) \psi \chi^{2} \mathrm{~d} x
\end{aligned}
$$

where $W:=\lambda-V$. Employing (1) and Cauchy-Schwarz inequality, we continue the calculations,

$$
\begin{aligned}
\nu\|\chi \nabla \psi\|_{L_{2}\left(M_{k, r}\right)}^{2} \leqslant & \frac{\nu}{2}\|\chi \nabla \psi\|_{L_{2}\left(M_{k, r}\right)}^{2}+2 \mu^{2} \nu^{-1}\|(\psi-k) \nabla \chi\|_{L_{2}\left(M_{k, r}\right)}^{2} \\
& +\int_{M_{k, r}}|W \| \psi|(\psi-k) \chi^{2} \mathrm{~d} x, \\
\|\chi \nabla \psi\|_{L_{2}\left(M_{k, r}\right)}^{2} \leqslant & 4 \mu^{2} \nu^{-2}\|(\psi-k) \nabla \chi\|_{L_{2}\left(M_{k, r}\right)}^{2} \\
& +2 \nu^{-1} \int_{M_{k, r}}|W \| \psi|(\psi-k) \chi^{2} \mathrm{~d} x .
\end{aligned}
$$

To estimate the last term in this inequality, we apply the Hölder inequality and 
Lemma 4.1,

$$
\begin{aligned}
\int_{M_{k, r}}|W||\psi|(\psi-k) \chi^{2} \mathrm{~d} x & \leqslant\|W\|_{L_{\frac{q}{2}}\left(M_{k, r}\right)}\left\|\psi|\psi-k| \chi^{2}\right\|_{L_{\hat{q}\left(M_{k, r}\right)}} \\
& \leqslant\|W\|_{L_{\frac{q}{2}}\left(M_{k, r}\right)}\left\|\left(|\psi-k|^{2}+k|\psi-k|\right) \chi^{2}\right\|_{L_{\hat{q}}\left(M_{k, r}\right)} \\
& \leqslant\|W\|_{L_{\frac{q}{2}}\left(B_{\frac{d}{4}}(\widetilde{a})\right)}\left\|3|\psi-k|^{2} \chi^{2}+2 k^{2}\right\|_{L_{\hat{q}}\left(M_{k, r}\right)} \\
& \leqslant \widehat{V}\left(3\|(\psi-k) \chi\|_{L_{2 \hat{q}}\left(M_{k, r}\right)}^{2}+2 k^{2}\left|M_{k, r}\right|^{\frac{1}{\tilde{q}}}\right)
\end{aligned}
$$

Now we employ inequality [12, Chapter I I, Section 2, (2.12)] that implies

$$
\begin{aligned}
\|(\psi-k) \chi\|_{L_{2 \hat{q}}\left(M_{k, r}\right)}^{2} \leqslant & C_{4}\left|M_{k, r}\right|^{2\left(\frac{1}{n}-\frac{1}{q}\right)}\|\nabla(\psi-v k) \chi\|_{L_{2}\left(M_{k, r}\right)}^{2} \\
\leqslant & C_{4} \Theta_{n}^{2\left(\frac{1}{n}-\frac{1}{q}\right)} r^{2\left(1-\frac{n}{q}\right)} \\
& \cdot\left(\|\chi \nabla \psi\|_{L_{2}\left(M_{k, r}\right)}^{2}+\|(\psi-v k) \nabla \chi\|_{L_{2}\left(M_{k, r}\right)}^{2}\right) .
\end{aligned}
$$

We substitute two last inequalities into (43) and take into account that the definition of $r_{1}$ and the inequality $r \leqslant r_{1}$ imply $3 C_{4} \Theta_{n}^{2\left(\frac{1}{n}-\frac{1}{q}\right)} r^{2\left(1-\frac{n}{q}\right)} \nu^{-1} \widehat{V} \leqslant \frac{1}{4}$. We also bear in mind that since $M_{k, r}=\varnothing$ for $k>1$, we can restrict our consideration to the case $-2 \leqslant k \leqslant 1$. In this case $|k| \leqslant 2$. By (43), (44) it leads us to the estimate

$$
\begin{aligned}
\|\chi \nabla \psi\|_{L_{2}\left(M_{k, r}\right)}^{2} & \leqslant\left(8 \mu^{2} \nu^{-2}+1\right)\|(\psi-k) \nabla \chi\|_{L_{2}\left(M_{k, r}\right)}^{2}+32 \nu^{-1} \widehat{V}\left|M_{k, r}\right|^{\frac{1}{\hat{q}}} \\
& \leqslant 9 \mu^{2} \nu^{-2}\|(\psi-k) \nabla \chi\|_{L_{2}\left(M_{k, r}\right)}^{2}+32 \nu^{-1} \widehat{V}\left|M_{k, r}\right|^{\frac{1}{\tilde{q}}}
\end{aligned}
$$

where we have used that $\frac{\mu}{\nu} \geqslant 1$. Now we take any $\delta \in(0,1)$ and assume that $\chi \equiv 1$ in $B_{r(1-\delta)}(a)$ and $|\nabla \chi| \leqslant 3(\delta r)^{-1}$ in $B_{r}(a)$. Then we obtain

$$
\|\nabla \psi\|_{L_{2}\left(M_{k, r(1-\delta)}\right)}^{2} \leqslant\left(81 \Theta_{n}^{\frac{2}{q}} \mu^{2} \nu^{-2} \delta^{-2} r^{-2\left(1-\frac{n}{q}\right)} \max _{\bar{M}_{k, r}}|\psi-k|^{2}+32 \nu^{-1} \widehat{V}\right)\left|M_{k, r}\right|^{\frac{1}{\tilde{q}}}
$$

This inequality means that the function $\psi$ belongs to the aforementioned class $\mathfrak{B}_{2}\left(B_{\frac{d}{4}}(\widetilde{a}), 1, C, \widetilde{C}, 1, \frac{1}{q}\right)$ with $C=81 \Theta_{n}^{\frac{2}{q}} \mu^{2} \nu^{-2}, \widetilde{C}=32 \nu^{-1} \widehat{V}$

Note that [12, Theorem 6.1] implies that the estimate (42) holds. To obtain explicit expressions for the exponent $\alpha$ and the constsnt $C_{14}$, one has to trace the dependence of the various constants trough the proof of [12, Theorem 6.1]. More precisely, one uses formula (6.36) in [12, Chapter II,Theorem 6.1], the choice $\delta_{0}=\frac{1}{2}$ given right after formula [12, Chapter II, (6.34)], formulae (6.24), (6.26) in the proof of [12, Chapter II, Lemma 6.3], formulae (6.16), (6.17) in the proof of [12, Chapter II, Lemma 6.2], and the formula established at the very end of the proof of [12, Chapter II, Lemma 3.8] for the constant $\beta$ which is introduced in [12, Chapter $\mathbb{I}$, Inequality (3.4)]. 
The final result of this section provides an upper bound on the $L_{2}$-norm of $\psi$, given the normalization (40).

Lemma 7.3. The estimate

$$
\|\psi\|_{L_{2}(\Omega)}^{2} \leqslant \frac{C_{18}}{|\lambda|}, \quad C_{18}:=\left(C_{1}+\frac{4 \mu}{r_{1}^{2}}\right)\left|\Omega_{0, \frac{d}{4}}\right|,
$$

holds true.

Proof. Let $\chi \in C^{\infty}(\bar{\Omega})$ be a cut-off function vanishing in $\bar{\Omega}_{0}$ and equalling one in $\Omega \backslash \Omega_{0, r_{1}}$. It is clear that $\psi \chi^{2} \in \mathcal{D}(\mathfrak{h})$. In view of this fact and the definition of $\psi$ we have

$$
\mathfrak{h}\left[\psi, \psi \chi^{2}\right]=\lambda\|\psi \chi\|_{L_{2}(\Omega)}^{2} .
$$

Direct calculations using the symmetry of $A_{i j}$ yield

$$
\begin{aligned}
\widetilde{\mathfrak{h}}\left[\nabla \psi, \nabla \psi \chi^{2}\right] & =\widetilde{\mathfrak{h}}[\chi \nabla \psi, \nabla \psi \chi]+\widetilde{\mathfrak{h}}[\chi \nabla \psi, \psi \nabla \chi] \\
& =\widetilde{\mathfrak{h}}[\nabla \chi \psi, \nabla \chi \psi]-\widetilde{\mathfrak{h}}[\psi \nabla \chi, \nabla \chi \psi]+\widetilde{\mathfrak{h}}[\psi \nabla \chi, \chi \nabla \psi] \\
& =\widetilde{\mathfrak{h}}[\nabla \chi \psi, \nabla \chi \psi] \widetilde{\mathfrak{h}}[\psi \nabla \chi, \psi \nabla \chi]
\end{aligned}
$$

We substitute this identity into (46) and obtain

$$
\mathfrak{h}[\psi \chi, \psi \chi]-\lambda\|\psi \chi\|_{L_{2}(\Omega)}^{2}=\widetilde{\mathfrak{h}}[\psi \nabla \chi, \psi \nabla \chi]
$$

The function $\psi \chi$ vanishes on $\Omega_{0}$ and this is why it belongs to the domain of the quadratic form associated with $\mathcal{H}_{0}$. The value of this quadratic form on $\psi \chi$ equals $\mathfrak{h}[\psi \chi, \psi \chi]$, and by assumptions (3c) and (3d) we obtain that $\lambda<0$ and $\mathfrak{h}[\psi \chi, \psi \chi] \geqslant 0$. These inequalities and (1) imply $|\lambda|\|\psi \chi\|_{L_{2}(\Omega)}^{2} \leqslant \mu\|\psi \nabla \chi\|_{L_{2}(\Omega)}^{2}$. We choose $\chi$ so that it takes values in $[0,1]$ and satisfies $|\nabla \chi| \leqslant 2 r_{1}^{-1}$ on $\Omega_{0, r_{1}} \backslash \Omega_{0}$. Hence,

$$
\begin{aligned}
\|\psi\|_{L_{2}\left(\Omega \backslash \Omega_{0, r_{1}}\right)}^{2} & \leqslant \frac{4 \mu}{r_{1}^{2}|\lambda|}\|\psi\|_{L_{2}\left(\Omega_{0, r_{1}}\right)}^{2} \\
\|\psi\|_{L_{2}(\Omega)}^{2} & \leqslant|\lambda|^{-1}\left(|\lambda|+\frac{4 \mu}{r_{1}^{2}}\right)\|\psi\|_{L_{2}\left(\Omega_{0, r_{1}}\right)}^{2} .
\end{aligned}
$$

We know by $(41)$ that $|\psi| \leqslant 1$ in $\Omega_{0, r_{1}}$. It also follows from the definition of $r_{1}$ that $\left|\Omega_{0, r_{1}}\right| \leqslant\left|\Omega_{0, \frac{d}{4}}\right|$. Substituting these estimates into (47) and employing the inequality $|\lambda|<C_{1}$ which is valid due to (10), we arrive at the statement of the lemma. 


\section{Proof of main results}

Proof of Theorem 2.2. Let $x_{+} \in \bar{\Omega}_{0}$ be the point where the function $\psi$ attains the maximum, i.e., $\psi\left(x_{+}\right)=1$. Such a point exists due to (40). Due to Lemma 7.1, there exist a point $x_{-}$in $\Omega_{0}$ such that $\psi\left(x_{-}\right)=0$. We connect the points $x_{+}$and $x_{-}$by an admissible cylinder and choose it as the domain $\Omega^{\prime}$. We fix the radius of the cylinder $\Omega^{\prime}$ setting it equal to

$$
r_{2}:=\min \left\{r_{1}\left(3 C_{14} C_{2}\right)^{-\frac{1}{\alpha}}, \frac{d}{8}, r_{0}\right\}
$$

where the constant $C_{2}$ is taken from Theorem 6.1 (Harnack inequality). We denote the bases of $\Omega^{\prime}$ by $S_{+}, S_{-}$(so that $x_{+} \in S_{+}$and $x_{-} \in S_{-}$). In this cylinder we introduce new coordinates: the arc length $s$ of the $C^{2}$-curve corresponding to $\Omega^{\prime}$ connecting $x_{+}$and $x_{-}$, and the coordinates on the cross-section. Since the cylinder is admissible, these coordinates are well-defined. We also observe that $\Omega^{\prime} \subseteq \widehat{\Omega}_{\frac{d}{8}}$.

Lemma 7.2 and the definition of $r_{2}$ imply that

$$
\psi(x) \geqslant 1-\left(3 C_{2}\right)^{-1} \text { for } x \in S_{+} \quad \text { and } \quad \psi(x) \leqslant\left(3 C_{2}\right)^{-1} \text { for } x \in S_{-} .
$$

We employ this inequality and the obvious estimate $\left|\frac{\partial \psi}{\partial s}\right| \leqslant|\nabla \psi|, x \in \Omega^{\prime}$, to obtain

$$
\begin{aligned}
\inf _{\Omega^{\prime}} \psi_{0} \cdot\left\|\nabla \frac{\psi}{\psi_{0}}\right\|_{L_{1}\left(\Omega^{\prime}\right)} & \geqslant \inf _{\widehat{\Omega}_{\frac{d}{8}}} \psi_{0} \cdot \int_{\Omega^{\prime}} \frac{\partial}{\partial s} \frac{\psi}{\psi_{0}} \mathrm{~d} x \\
& =\inf _{\widehat{\Omega}_{\frac{d}{8}}} \psi_{0} \cdot\left(\int_{S_{+}} \frac{\psi}{\psi_{0}} \mathrm{~d} S_{+}-\int_{S_{-}} \frac{\psi}{\psi_{0}} \mathrm{~d} S_{-}\right) \\
& \geqslant S_{0}\left(\frac{\inf _{\widehat{\Omega}_{\frac{d}{8}}} \psi_{0}}{\sup _{\widehat{\Omega}_{\frac{d}{8}}} \psi_{0}}\left(1-\left(3 C_{2}\right)^{-1}\right)-\left(3 C_{2}\right)^{-1}\right) \\
& \geqslant \frac{S_{0}\left(2 C_{2}-1\right)}{3 C_{2}^{2}} \\
& \geqslant \frac{S_{0}}{3 C_{2}},
\end{aligned}
$$

where $S_{0}:=\left|S_{+}\right|=\left|S_{-}\right|$. Recall that the cylinder $\Omega^{\prime}$ is defined with the help of a curve connecting the points $x_{-}$and $x_{+}$. Let $\ell$ be the length of the $C^{2}$-curve connecting $x_{-}$and $x_{+}$. To estimate the volume of $\Omega^{\prime}$ we will need the following auxiliary

Lemma 8.1. The equality $\left|\Omega^{\prime}\right|=S_{0} \ell$ holds true. 
Proof. Let $R(s)$ be the vector-function describing the $C^{2}$-curve connecting $x_{-}$ and $x_{+}$, where $s$ is the arc length, $T(s)$ be the tangential vector to this curve, and $N_{i}(s), i=1, \ldots, n-2$ be the continuously differentiable vectors orthogonal to $T(s)$. We assume that $N_{i}$ are orthonormalized, so, the vectors $T$ and $N_{i}(s)$ form a Frenet frame attached to the curve. The vectors $N_{i}$ form an orthonormalized basis in the $(n-1)$-dimensional disk attached to the same point of the curve as $N_{i}$. As the corresponding Cartesian coordinates $y$ we choose the ones associated with the vectors $N_{i}$. As a result we have

$$
x=R(s)+\sum_{i=1}^{n-1} y_{i} N_{i}(s) .
$$

By $J(s, y)$ we denote the Jacobian

$$
J(s, y)=\frac{D(x)}{D(s, y)}=\operatorname{det} M_{J}, \quad \text { where } \quad M_{J}:=\left(\begin{array}{c}
T(s)+\sum_{i=1}^{n-1} y_{i} N_{i}^{\prime}(s) \\
N_{1}(s) \\
\vdots \\
N_{n-1}(s)
\end{array}\right)
$$

Since the vectors $T$ and $N_{i}$ are orthonormalized, the matrix $\left(T, N_{1}, \ldots, N_{n-1}\right)$ is unitary and up to a renumbering of $N_{i}$ we can assume that its determinant equals one. Hence, if we multiply $M_{J}$ by this matrix, we do not change the value of $J$. It gives

$$
J(s, y)=\operatorname{det}\left(\begin{array}{ccccc}
1+\sum_{i=1}^{n-1} y_{i} k_{i}(s) & * & * & \ldots & * \\
0 & 1 & 0 & \ldots & 0 \\
0 & 0 & 1 & \ldots & 0 \\
\vdots & \vdots & \vdots & \ddots & \vdots \\
0 & 0 & 0 & \ldots & 1
\end{array}\right)=1+\sum_{i=1}^{n-1} y_{i} k_{i}(s)
$$

where the symbol $*$ indicates unspecified functions, $k_{i}(s)=\left(T(s), N_{i}^{\prime}(s)\right)_{\mathbb{R}^{n}}$. Since for each $s$ the vectors $\left(T, N_{1}, \ldots, N_{n-1}\right)$ form a basis the map $s \mapsto J(s, y)$ never vanishes. As $J(s, y)=1$ for $y=0$, we conclude that $J(s, y)$ is a positive function. Employing this fact and (49), we can calculate the volume of $\Omega^{\prime}$ :

$$
\left|\Omega^{\prime}\right|=\int_{0}^{\ell} \mathrm{d} s \int_{|y|<r_{2}} J(s, y) \mathrm{d} y=\left|S_{0}\right| \ell+\int_{0}^{\ell} \mathrm{d} s \sum_{i=1}^{n-1} k_{i}(s) \int_{|y|<r_{2}} y_{i} \mathrm{~d} y .
$$

By parity arguments $\int_{|y|<r_{2}} y_{i} \mathrm{~d} y=0$. Together with the previous identity this completes the proof of the auxiliary lemma.

Now we continue the proof of Theorem 2.2. For this purpose we substitute the proved identity $\left|\Omega^{\prime}\right|=S_{0} \ell$ and (48)into (14), and arrive at the estimate

$$
\lambda-\lambda_{0} \geqslant \frac{S_{0}^{2} \nu}{9 C_{2}^{2}\left|\Omega^{\prime}\right|\|\psi\|_{L_{2}(\Omega)}^{2}} \geqslant \frac{S_{0} \nu}{9 C_{2}^{2} L\|\psi\|_{L_{2}(\Omega)}^{2}},
$$


where we have used that $\ell \leqslant L$. The $(n-1)$-dimensional volume of the discs $S_{+}, S_{-}$equals $S_{0}=\Theta_{n-1} r_{2}^{n-1}$. We substitute this identity and (45) into (50) and arrive at (5). In this inequality we changed the notations, namely, we denoted $c_{1}:=r_{2}, c_{2}:=C_{13}, c_{3}:=C_{14}, c_{4}:=C_{15}, c_{5}:=C_{16}, c_{6}:=C_{17}, c_{7}:=C_{10}$, $c_{8}:=C_{5} \frac{C_{7}}{p}, c_{9}:=C_{12} \frac{\hat{q}}{p}$.

Proof of Theorem 2.4. Let us prove the estimate (6). The parameter $L$ appears only explicitly in the right hand side of (5), and also in the definition of $c_{1}$. We also observe that $\left|\Omega_{0, \frac{d}{4}}\right|$ is bounded by $\Theta_{n} L^{n}$. The constants $c_{i}, i=2, \ldots, 10$ are independent of $L$. It follows from (38) and the formula for $c_{3}$ that $c_{2}>1$, $c_{3}>1$. Hence, for $L$ large enough $c_{1}=r_{1}\left(3 c_{3} c_{2}^{\frac{8 L}{d}}\right)^{-\frac{1}{\alpha}}$. We substitute this identity into (5) and arrives at (6), where $c_{11}:=8 d^{-1}\left(1+(n-1) \alpha^{-1}\right) \ln c_{2}>0$.

Proof of Theorem 2.5. It is clear that for $\widehat{V}$ small enough all the constants remain bounded from above and below. We also note that $\left\|V^{-}\right\|_{L_{\frac{q}{2}}\left(\Omega_{0}\right)}$ is small, too. The mentioned facts imply (7).

Proof of Theorem 2.6. In the case considered the constants $\alpha, c_{3}-c_{6}$ remain constant and depend on $n, q$, and $\frac{\mu}{\nu}$. The constants $c_{7}-c_{10}$ satisfy the relations

$$
c_{7}^{-1} \leqslant C_{19} \nu^{-\frac{1}{2}}, \quad c_{8} \leqslant C_{20} \nu^{-2}, \quad c_{9} \leqslant C_{21} \nu^{-1} .
$$

where $C_{i}=C_{i}(n, q, d, \widehat{V}), i=19,20,21$. Hence,

$$
c_{2} \leqslant C_{22} \nu^{-\frac{\hat{q}}{p-\hat{q}}}\left(C_{23} \log _{\frac{p}{\hat{q}}}^{2} \nu\right)^{C_{24} \nu^{-\frac{1}{2}}}, \quad r_{1}=C_{25} \nu^{\frac{q}{2(q-n)}}, \quad c_{1}=C_{26} \nu^{\frac{q}{2(q-n)}} c_{2}^{-\frac{8 L}{\alpha d}}
$$

where $C_{i}=C_{i}(n, q, d, \widehat{V}), i=22, \ldots, 26, C_{24}>0, C_{25} \neq 0, C_{26} \neq 0$. We substitute these relations into (5) and obtain (8).

Proof of Theorem 2.7. The proof of (9) is more complicated in comparison with the previous proof. Namely, in this case the estimates (51) for $c_{7}, c_{8}$, remain true, where $C_{i}=C_{i}(n, q, d, \mu, \widehat{V})$. The estimates for $c_{9}$ and $c_{2}$ read as follows,

$$
c_{9} \leqslant C_{27} \nu^{-1} \log _{\frac{p}{\hat{q}}}^{2} \nu, c_{2} \leqslant\left(C_{28} \nu^{-1} \log _{\frac{p}{\tilde{q}}}^{2} \nu\right)^{C_{29} \nu^{-\frac{1}{2}}}
$$

$C_{i}=C_{i}(n, q, d, \mu, \widehat{V}), i=27,28,29$. The main difference with the previous case is that now the constants $c_{3}-c_{6}$ and $\alpha$ depend on $\nu$ in a singular way. Namely,

$$
c_{6} \leqslant C_{30} \nu^{-1}, \quad c_{5} \leqslant C_{31} \nu^{-\frac{q n}{q-n}}, \quad c_{4} \leqslant C_{32} \nu^{-\frac{2(n-1) q}{q-n}} .
$$

where $C_{i}=C_{i}(n, q, \mu), i=30,31,32$. Thus, $\alpha=-\log _{4}\left(1-2^{-c_{4}}\right)$, and $C_{33} \exp \left(-C_{34} \nu^{-\frac{2(n-1) q}{q-n}}\right) \geqslant \alpha \geqslant C_{35} \exp \left(-C_{34} \nu^{-\frac{2(n-1) q}{q-n}}\right), \quad c_{3} \leqslant C_{37} \exp \left(C_{38} \nu^{-\frac{2(n-1) q}{q-n}}\right)$, 
where $C_{i}=C_{i}(n, q, \mu), i=34,36,37, C_{34}>0, C_{38}>0, C_{33}$ and $C_{35}$ are some absolute constants. We also observe that $r_{1} \sim \nu^{\frac{q}{2(q-n)}}, c_{1}=r_{1}\left(3 c_{3} c_{2}^{\frac{8 L}{d}}\right)^{-\frac{1}{\alpha}}$. Bearing in mind the obtained relations, we estimate the right hand side of (5) from below that gives the following inequality

$$
\begin{aligned}
\lambda-\lambda_{0} & \geqslant\left|\Omega_{0, \frac{d}{4}}\right|^{-1} C_{39}|\lambda| L^{-1} \nu c_{1}^{n-1} r_{1}^{2} c_{2}^{-\frac{8 L}{d}} \\
& \geqslant C_{39}|\lambda| L^{-n-1} \nu r_{1}^{n+1} 3^{-\frac{n-1}{\alpha}} c_{3}^{-\frac{n+1}{\alpha}} c_{2}^{-\frac{8 L n}{\alpha d}} \\
& \geqslant C_{40}|\lambda| L^{-n-1} \nu^{1+\frac{q(n+1)}{2(q-n)}} \exp \left(-C_{41} \alpha^{-1} \nu^{-\frac{2(n-1) q}{q-n}}\right)\left(C_{28} \nu^{-1} \log _{\frac{p}{\tilde{q}}}^{2} \nu\right)^{-\frac{8 C_{24} L n}{\alpha d \sqrt{\nu}}}
\end{aligned}
$$

where $C_{39}=C_{39}(n, d, \mu), C_{40}=C_{40}(n, d, \mu, \widehat{V}), C_{41}=C_{41}(n, q, \mu)$. We substitute into the obtained inequality the estimate for $\alpha$ that implies (9).

Acknowledgments. D.B. was partially supported by RFBR, by the Federal Task Program of the Ministry of Education and Science of Russia (contract no. 02.740.11.0612), and by FCT, project PTDC/ MAT/ 101007/2008. Both authors were partially supported through the project "Spektrale Eigenschaften von zufälligen Schrödingeroperatoren und zufälligen Operatoren auf Mannigfaltigkeiten und Graphen" within the Emmy-Noether-Programme of the Deutsche Forschungsgemeinschaft.

\section{References}

[1] Borisov, D., Asymptotic behaviour of the spectrum of a waveguide with distant perturbations. Math. Phys. Anal. Geom. 10 (2007), 155 - 196.

[2] Borisov, D., Distant perturbations of the Laplacian in a multi-dimensional space. Ann. Henri Poincaré 8 (2007), 1371 - 1399.

[3] Davies, E. B., Heat Kernels and Spectral Theory. Cambridge: Cambridge University Press 1989.

[4] Duclos, P. and Exner, P., Curvature-induced bound states in quantum waveguides in two and three dimensions. Rev. Math. Phys. 7 (1995), 73 - 102.

[5] Gilbarg, D. and Trudinger, N., Elliptic Partial Differential Equations of Second Order. Berlin: Springer 1977.

[6] Harell, E., On the rate of asymptotic eigenvalue degeneracy. Comm. Math. Phys. 60 (1978), $73-95$.

[7] Kirsch, W. and Simon, B., Universal lower bounds of eigenvalue splittings for one dimensional Schrödinger operators. Comm. Math. Phys. 97 (1985), $453-460$.

[8] Kirsch, W. and Simon, B., Comparison theorems for the gap of Schrödinger operators. J. Funct. Anal. 75 (1987), $396-410$. 
[9] Kolmogorov, A. N. and Fomin, S. V., Introductory Real Analysis. Transl. from second Russian edition, ed.: R. A. Silverman, corrected reprinting. New York: Dover 1975.

[10] Kondej, S. and Veselić, I., Lower bounds on the lowest spectral gap of singular potential Hamiltonians. Ann. Henri Poincaré 8 (2007), 109 - 134.

[11] Kuchment, P., The mathematics of photonic crystals. In: Mathematical Modeling in Optical Science (eds.: G. Bao et al.). Frontiers Appl. Math. 22. Philadelphia (PA): SIAM 2001, pp. $207-272$.

[12] Ladyzhenskaya, O. A. and Uraltseva, N. N., Lineinye i Kvazilineinye Uravneniya Ellipticheskogo Tipa (in Russian). Second rev. edition. Moscow: Nauka 1973.

[13] Ma, L. and Liu, B., Convexity of the first eigenfunction of the drifting Laplacian operator and its applications. New York J. Math. 14 (2008), 393 - 401.

[14] Simon, B., Semiclassical analysis of low lying eigenvalues. II. Tunneling. Ann. Math. 120 (1984), 89 - 118.

[15] Simon, B., Semiclassical analysis of low lying eigenvalues. III. Width of the ground state band in strongly coupled solids. Ann. Physics 158 (1984), $415-420$.

[16] Simon, B., Semiclassical analysis of low lying eigenvalues. IV. The flea on the elephant. J. Funct. Anal. 63 (1985), 123 - 136.

[17] Singer, I. M., Wong, B., Yau, S.-T. and Yau, S. S.-T., An estimate of the gap of the first two eigenvalues in the Schrödinger operator. Ann. Scuola Norm. Sup. Pisa Cl. Sci. (4) 12 (1985), $319-333$.

[18] Vogt, H., A lower bound on the first spectral gap of schrödinger operators with Kato class measures. Ann. Henri Poincaré 10 (2009), 395 - 414.

[19] Yau, S.-T., An estimate of the gap of the first two eigenvalues in the Schrödinger operator. In: Lectures on Partial Differential Equations (eds.: S.-Y. A. Chang et al.). New Stud. Adv. Math. 2. Somerville (MA): Int. Press 2003, pp. $223-235$.

[20] Yau, S.-T., Gap of the first two eigenvalues of the Schrödinger operator with nonconvex potential. Mat. Contemp. 35 (2008), $267-285$.

Received April 21, 2011; revised February 20, 2012 